\title{
ACCELERATION OF JACCARD'S INDEX ALGORITHM FOR TRAINING TO TAG DAMAGE ON POST-EARTHQUAKE IMAGES
}

\author{
A Thesis \\ presented to \\ the Faculty of California Polytechnic State University, \\ San Luis Obispo
}

\author{
In Partial Fulfillment \\ of the Requirements for the Degree \\ Master of Science in Computer Science
}

by

Kyle Mulligan

June 2018 
(C) 2018

Kyle Mulligan

ALL RIGHTS RESERVED 


\section{COMMITTEE MEMBERSHIP}

TITLE:

AUTHOR:

DATE SUBMITTED: June 2018

COMMITTEe CHAIR: Maria Pantoja, Ph.D.

Professor of Computer Science

COMMITTEE MEMBER: Chris Lupo, Ph.D.

Professor of Computer Science

COMMITTEE MeMBER: Franz Kurfess, Ph.D.

Professor of Computer Science 


\begin{abstract}
Acceleration of Jaccard's Index Algorithm for Training to Tag Damage on

Post-Earthquake Images
\end{abstract}

Kyle Mulligan

There are currently different efforts to use Supervised Neural Networks (NN) to automatically label damages on images of above ground infrastructure (buildings made of concrete) taken after an earthquake. The goal of the supervised NN is to classify raw input data according to the patterns learned from an input training set. This input training data set is usually supplied by experts in the field, and in the case of this project, structural engineers carefully and mostly manually label these images for different types of damage. The level of expertise of the professionals labeling the training set varies widely, and some data sets contain pictures that different people have labeled in different ways when in reality the label should have been the same. Therefore, we need to get several experts to evaluate the same data set; the bigger the ground truth/training set the more accurate the NN classifier will be. To evaluate these variations among experts, which can be considered equal to the task of evaluating the quality of the expert, using probabilistic theory we first need to implement a tool able to compare different images classified by different experts and apply a certainty level to the experts tagged labels. This master's thesis implements this comparative tool. We also decided to implement the comparative tool using parallel programming paradigms since we foresee that it will be used to train multiple young engineering students/professionals or even novice citizen volunteers (trainees) during after-earthquake meetings and workshops. The implementation of this software tool involves selecting around 200 photographs tagged by an expert with proven accuracy (ground truth) and comparing them to files tagged by the trainees. The trainees are then provided with instantaneous feedback on the accuracy of their damage assess- 
ment. The aforementioned problem of evaluating trainee results against the expert is not as simple as comparing and finding differences between two sets of image files. We anticipate challenges in that each trainee will select a slightly different sized area for the same occurrence of damage, and some damage-structure pairs are more difficult to recognize and tag. Results show that we can compare 500 files in 1.5 seconds which is an improvement of $2 \mathrm{x}$ faster compared to sequential implementation. 


\section{ACKNOWLEDGMENTS}

Thanks to:

- Dr. Maria Pantoja, for being an excellent advisor and guiding me through my thesis

- Dr. Chris Lupo for taking the time to be on my committee and providing constructive feedback

- Dr. Franz Kurfess for taking the time to be on my committee and providing constructive feedback

- My family, for supporting me in my scholastic endeavors

- Mike Ryu, for providing some test file generating code and for being my reliable rubber duck

- Erin Wint for encouraging me to work on this thesis when I didn't want to and for helping me explain the work to people who would ask about it

- Katherine Flitsch for helping edit my thesis at the writing center

- Andrew Guenther, for uploading this Latex template 


\section{TABLE OF CONTENTS}

Page

LIST OF TABLES . . . . . . . . . . . . . . . . . . ix

LIST OF FIGURES . . . . . . . . . . . . . . . . . . . . . CHAPTER

1 Introduction . . . . . . . . . . . . . . . . . 1

2 Related Work . . . . . . . . . . . . . . . . . . . 6

2.1 Post-Earthquake Reconnaissance Techniques . . . . . . . . . . . . . 6

2.1.1 3D Laser Scanning . . . . . . . . . . . . . . 6

2.1.2 Close Range Photogrammetry With Tablet Technology in PostEarthquake Scenario . . . . . . . . . . . . . . 7

2.1.3 Visual Data Classification in Post-Event Building Reconnaissance 9

3 System Design . . . . . . . . . . . . . . . . . . . . . . . 11

3.1 System Overview . . . . . . . . . . . . . . . . . . . . . 11

3.2 Photo Tagging Tool for Rapid and Detailed Post-Earthquake Structural Damage Identification . . . . . . . . . . . . . . . 13

3.3 Early Approaches . . . . . . . . . . . . . . . . 16

3.3.1 Language Decision . . . . . . . . . . . . . . . . . . 17

3.3.2 Rectangle Comparison . . . . . . . . . . . . . . 17

$3.4 \mathrm{C}++\ldots \ldots \ldots \ldots \ldots \ldots$

3.5 Jaccard's Index . . . . . . . . . . . . . . . . . . . . . 19

4 Implementation . . . . . . . . . . . . . . . . . . . . . 22

4.1 Class Models . . . . . . . . . . . . . . . . . 22

4.1 Image Class . . . . . . . . . . . . . . . . . . 22

4.1 .2 Rectangle Class . . . . . . . . . . . . . . . . . . . . . 24

4.2 System Details . . . . . . . . . . . . . . . 25

4.2.1 Reading Input Data . . . . . . . . . . . . . . 25

4.2 .2 Image Comparison . . . . . . . . . . . . . 28

4.2 .3 Output Files. . . . . . . . . . . . . . . . 46

5 Testing, Evaluation, and Discussion . . . . . . . . . . . . 48

5.1 Testing . . . . . . . . . . . . . . . . . . 48 
5.1 .1 Overview . . . . . . . . . . . . . . . . . . . 48

5.1 .2 Testing Platform . . . . . . . . . . . . . . . . . . 49

5.1 .3 Data Files . . . . . . . . . . . . . . . . . . . . 50

5.1 .4 Test File Generator . . . . . . . . . . . . . . . . . . . 51

5.1 .5 Timing . . . . . . . . . . . . . . . . . . . 52

$5.1 .6 \quad$ Expert Files . . . . . . . . . . . . . . . . . 54

5.1 .7 Profiling and Program Validation . . . . . . . . . 56

5.2 Evaluation . . . . . . . . . . . . . . . . . . 56

5.2 .1 Single-File Timings . . . . . . . . . . . . . . . . . . 57

5.2 .2 Multi-File Timings . . . . . . . . . . . . . . . . . . 57

5.2 .3 High Performance Computer Timings . . . . . . . . . . . 58

5.2 .4 Expert File Comparisons . . . . . . . . . . . . . . . . . 61

5.2.5 Profiling and Program Validation . . . . . . . . . . . 61

5.3 Discussion . . . . . . . . . . . . . . . . . . . . . . . . . . . 64

5.3 .1 Single-File Timings . . . . . . . . . . . . . . . . . . . 64

5.3 .2 Multi-File Timings . . . . . . . . . . . . . . . . 66

5.3.3 High Performance Computer Timings . . . . . . . . . . . 68

5.3 .4 Expert File Comparisons . . . . . . . . . . . . . . . 68

5.3.5 Profiling and Program Validation . . . . . . . . . . . 72

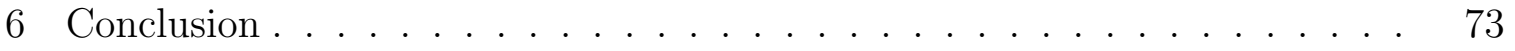

7 Future Work . . . . . . . . . . . . . . . . . . . . . 75

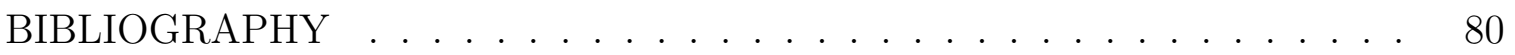
APPENDICES

A Repository ............................. 82 


\section{LIST OF TABLES}

Table

5.1 Results of High Performance Computer doing single file tests . . . . 58

5.2 Results of High Performance Computer doing multiple file tests . . 58

5.3 Results using Ezra1's file as expert . . . . . . . . . . . . 61

5.4 Results using Ezra2's file as expert . . . . . . . . . . . . 62

5.5 Results using Ana's file as expert . . . . . . . . . . . 62

5.6 Results using Erika's file as expert . . . . . . . . . . . . 62

5.7 Results using John's file as expert . . . . . . . . . . . . 63 


\section{LIST OF FIGURES}

Figure

2.1 Examples of laser scan data from Japan and Chile following the earthquakes and tsunamis. (a) Topographic mapping in Onagawa, Japan following the tsunami destruction. (b) Steel frame structure in Yuriage, Japan damaged by tsunami impact. (c) Damaged walls from pressurization at a concrete ware- house in Onagawa, Japan. Tsunami damage to (d) the City Hall and (e) the Capital 100 Hotel in Rikuzentakata, Japan. (f) Deformation analysis of a concrete wall blow-in at the Gamou Wastewater treatment plant. (g) Damage to a concrete deck at a port facility in Talcahuano, Chile.[11] . . . . .

2.2 (a) Multi-image configuration. (b) Cameras geometry configuration

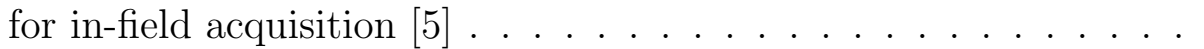

2.3 Samples of ground truth (labeled) images: (left) collapse and (right) spalling [17]........................... 10

3.1 System overview diagram . . . . . . . . . . . . . . . . . . 12

3.2 Reinforced concrete building image tagging module (yellow boxes and labels added) [4]. . . . . . . . . . . . . . . . . . . 14

3.3 Example of Jaccard's index applied to rectangle areas . . . . . . . . 20

4.1 Flow diagram for implementation of the Fast Feedback Tool . . . . 23

4.2 Flow diagram for the getImgData function . . . . . . . . . . 26

4.3 Flow diagram for imageCompSerial function . . . . . . . . . . . 29

4.4 Flow diagram for rectangleCompSerial function . . . . . . . . . . 30

4.5 Flow diagram for traineeRectComp function . . . . . . . . . . . . 31

4.6 Different ways in which rectangles may intersect: A. Top and left edge of intersection from blue rectangle right and bottom edge from red rectangle; B. All intersection edges from same rectangle; C. Three edges from one rectangle; D. No intersection area . . . . . . . . . . 37

4.7 Flow diagram for imageCompParallel function . . . . . . . . . . . 39

4.8 Flow diagram for rectangleCompareParallel function . . . . . . . . 41

5.1 Results of High Performance Computer timing evaluations of Single File comparisons plotted against the number of threads used. 
5.2 Results of High Performance Computer timing evaluations of Multiple File comparisons plotted against the number of threads used. 60

5.3 Execution profiling INCLUDING the rectangle-to-rectangle parallel portions. Items of interest underlined in red. . . . . . . . . . . 63

5.4 Execution profiling EXCLUDING the rectangle-to-rectangle parallel portions. Items of interest underlined in red. . . . . . . . . . . . 64

5.5 Two rectangles of the same size and shape offset by 6 pixels in the $\mathrm{X}$ and $\mathrm{Y}$ direction with a Jaccard's index score of $0.625 \ldots \ldots$

$5.6 \quad$ Jaccard's index scores as the overlap of the rectangles in figure 5.5 decreases. Note that the score falls off dramatically even for a small amount of misalignment . . . . . . . . . . . . . . . .

7.1 Example of two different ways to mark a diagonal crack in a wall. Which method is better and can both be used in future work? . . . 76 


\section{Chapter 1}

\section{INTRODUCTION}

Immediately following a major earthquake, many buildings in the affected area will be left in a state of minor to extreme damage. Determining the extent of the damage that these buildings have sustained will be of great importance in the days following the disaster, as victims need to find safe places to stay, and as those who were injured are treated in critical buildings such as hospitals. Determining the safety of these buildings in a timely manner is of further importance when there are concerns about aftershocks occurring or severely damaged buildings collapsing without warning. Establishing which buildings need attention and which ones need to be evacuated is a high priority during the aftermath of a major disaster.

After a major disaster, many images of the affected area will be compiled by civil/structural engineering reconnaissance teams and uploaded to the Internet via media/social media platforms by citizens, amateur photographers, and journalists. These images can be tagged with the building damages by seasoned structural engineers who have specific experience in post-earthquake reconnaissance. But this process can be very time consuming, considering the vast amount of data and the limited number of qualified engineers.

In the immediate aftermath of the Haiti earthquake in 2010, hundreds of medical volunteers alone flocked to the affected area to provide aide to the citizens affected by the disaster [7]. The number of volunteers probably ranges into the thousands in situations like this, and not all volunteers have to be specially trained experts in order to contribute to the relief effort. The training that will eventually be provided by this project could encourage more civilians to volunteer their time and effort when a disaster such as the Haiti earthquake occurs. 
It is not necessary that the individuals tagging damage be post-earthquake reconnaissance experts. Training engineers to tag for damage and structure is difficult and unstandardized. The work done by Dr. Behrouzi and Dr. Pantoja in [4] aims to provide a consistent approach that facilitates the training of young engineering students/professionals or even novice citizen volunteers (trainees) by standardizing the way they identify and tag specific damage-structure pairs. Implementation of this software tool involves selecting around 200 photographs tagged by experts with proven accuracy (ground truth). Individuals then compare the files tagged by the trainees to the expert-tagged files. This feedback method is time consuming as it requires manual grading. By automating the grading process, this project provides trainees with immediate feedback on the accuracy of their damage assessment. "Based on Formative Assessment pedagogical theory, prompt and accurate feedback is very important to give students" [4].

After an earthquake thousands of pictures get uploaded to the Internet by citizens living in the affected areas or by professional, first-responder organizations. These pictures are later carefully tagged for different damages to create reports used by reconnaissance teams that evaluate the damages and give recommendations about demolishing, improvements, retrofitting and others. The problem is that reconnaissance teams ideally should be deployed within a week of the earthquake event, but manually tagging these pictures takes much longer than that. To facilitate the automatic tagging of the images in previous work we created a NN [4] to classify these damages.

The computer vision algorithm that was used is a supervised NN. Supervised algorithms learn from previously, mainly manually, tagged input images. all possible combinations of damages must be identifiable for structures built using concrete as the building material. This is around 20 different types of damages and around around 10 different types of structures. These damage/structure pairs translate to around 
200 different combinations (some of the combinations are not possible).

The NN needs minimum 200 pictures for each damage/structure pair. The 200 images, is the minimum requirement, and the general consensus is that more would be better. Therefore 40000 images from one expert are needed to train a NN to classify damages in concrete civil infrastructures. Unfortunately there is no consensus among structural engineers about what is the best way to label an image; by this we mean that an expert can label a damage-structure a certain way and a different expert will choose completely different damage pairs. This problem remains even if there is a fixed set of labels the experts can choose from and provide a uniform set of damages and structures to select from. To account for different experts opinions and make the NN useful and generally accepted, several different structural engineers will need to be asked to label the same group of pictures; and the size of the corpus increases dramatically since the requirements will be 40,000 multiplied by the number of experts. To get reasonable results a minimum of five experts are needed, for a total of around 200,000 images. Gathering images from experts is a very slow process, much slower than what initially was expected; expert time is expensive and obtaining the amount of required labeled data may take years [8]. Reducing the number of images needed for the training while still giving an accurate output for the labeled damages is a major goal to make this project feasible. To accomplish that goal we need to answer the following questions:

1. Can the quality of the expert that tags the pictures for the training be consistently and fairly evaluated? Some of the experts have been observed in the process of tagging the pictures and there is clearly uncertainty on the labels, sometimes because the image presents an incomplete picture of the building or because the expert cannot decide between different values for the tags that are so close visually that the expert cant decide if is one or the other. How to 
evaluate this certainty of the expert is explained in Chapter 2 .

2. Can the number of potential labels be reduced? There are potentially 200 different label pairs, if 200 images per pair are needed, that means the algorithm needs 40000 images tagged by experts. This is not only a big data set, the problem is also that it takes a long time to find these images and then label them, around 40 min per image. How to reduce the damage/structure set is explained in Chapter 7.

To answer these questions we decided to implement the following steps:

1. Get a small set of images for which the labeled locations for the structuredamage pairs are known.

2. Get several structural engineers to label the same set.

3. Compare the labels damage-structure pairs for the different sets. This is the main purpose of this master thesis to develop a comparative tool that can provide statistics about the quality of the expert.

4. Assign, based on the results of this comparison, a basic assessment for the quality of the expert.

5. Modify the original NN to instead of giving an output it gives a probability distribution that will indicate the certainty of the automatically generated labels. The idea is that if many different experts labeled this kind of structure-damage consistently in the same way then the certainty should be high and the output distribution should be small; while if the damage-structure has only been tagged by one of the experts and the expert has been evaluated as not being very accurate in Step 3, The certainty should be low and the output distribution should be larger. 
In this master's thesis a fundamental algorithm is developed that will allow regions labeled by different structural engineers and the sets of images to be compared in order to get a measure of the uncertainty of the experts. This is called the Fast Feedback Tool. The algorithm may need to be implemented in parallel for use in conferences and workshops where more than 100 structural engineers can be trained to label images or for use in giving feedback to the neural network.

This thesis contributes substantially to the current state of earthquake reconnaissance. By giving trainees immediate feedback on the images that they tag, the training process is expedited. This acceleration of the training process allows for quicker tagging of images from the affected area, allowing for buildings to be assessed for structural soundness in a much shorter time period. This will greatly increase the safety of those in the affected area as they will receive information about what buildings are safe much sooner. Additionally, the training process can be standardized once a good ground truth set of images and solutions is developed, making the gathering of more images with more damage tags far more practical for the use of training the neural network previously developed by Dr. Pantoja and Dr. Behrouzi. 
Chapter 2

\section{RELATED WORK}

\subsection{Post-Earthquake Reconnaissance Techniques}

Currently several different methods for performing post-earthquake reconnaissance are in use. While the following techniques are very different in method and application, they all require a specially trained team to be deployed in the affected area in order to gather the necessary data. This results in the reconnaissance being very time.

\subsubsection{D Laser Scanning}

Some teams utilize 3D Laser Scanning devices to perform reconnaissance on affected areas. The data that can be acquired from these devices is highly accurate and provides significant raw data for later analysis. These laser scanners are capable of gathering precise geometrical data with a high degree of detail. This data can then be used to form a 3D model of the scanned area (usually buildings) in order to determine what engineering practices succeeded/failed in the building's structure. In-depth investigations of the effects of the disaster on a specific building can be performed using the 3D data acquired from these scans. There are three types of scanners, each with its own strengths and weaknesses. Figure 2.1 shows digitally generated models of buildings that have been laser scanned in a post-event scenario.

- ALS

Airborne laser scanning (ALS) systems have several advantages for reconnaissance. They can acquire data fast and cover large sections of terrain.

- STLS 
Static Terrestrial Laser Scan (STLS) systems are typically mounted to a tripod and are a slower method of collecting data for a large area. However, STLS offers improved resolution and accuracy for analyses of small sites or buildings compared to airborne systems.

\section{- MTLS}

Mobile Terrestrial Laser Scan (MTLS) systems can acquire data fast from a moving vehicle. Geo-referencing can be completed directly with the combination of components for the scanner. However, scan extents are limited to the roadway $+/-100 \mathrm{~m}$, and scanning may not be feasible if the roadway is significantly damaged and nonnavigable.[11]

While these scanners are very powerful tools for post-earthquake analysis, they have many drawbacks that make them a poor solution for the problems presented in this paper. The scanners must be deployed and used by highly trained specialists. The scanners are large and cumbersome, making them difficult to bring to the affected areas for deployment, meaning that the area that can be scanned by these devices is generally small in comparison to the total area affected by the disaster. Furthermore, if the scanning cannot be done from a close enough distance, the data acquired from the scanners becomes less detailed. Overall, 3D laser scanning tools are not a good option for determining building safety in the immediate aftermath of an earthquake, due to the time constraints and the need to analyze a large area.

\subsubsection{Close Range Photogrammetry With Tablet Technology in Post-Earthquake Scenario}

This project [5] is most similar to the work being done by Dr. Pantoja and Dr. Behrouzi. Utilizing consumer electronics such as tablets, smart-phones, and other devices with similar camera capabilities, the authors developed an artificial intelligence algorithm that uses images taken of buildings to digitally recreate the structures as 3D models that later can be analyzed by experts. The data for this reconnaissance method can be acquired in a very similar fashion to that of Dr. Pantoja and Dr. 


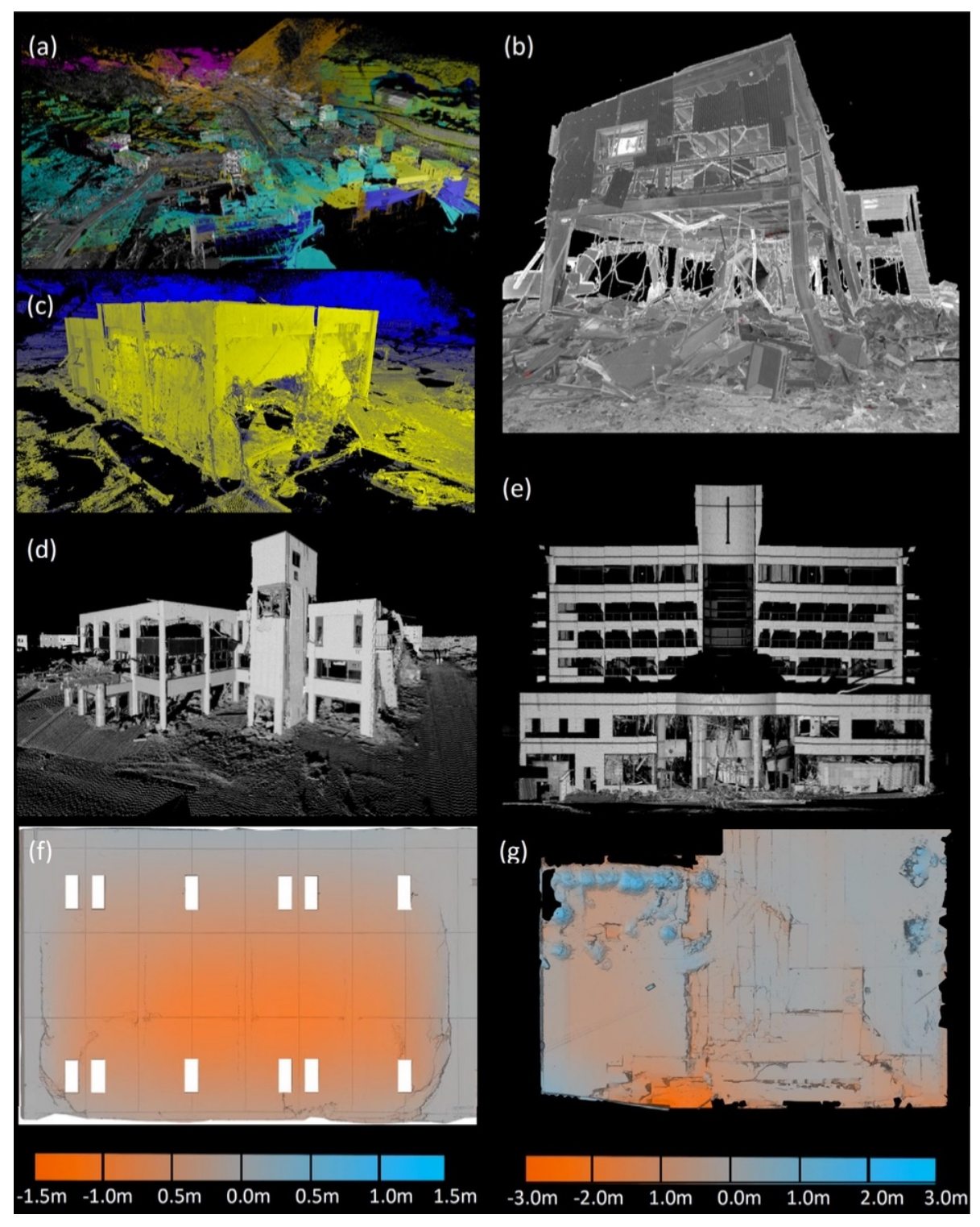

Figure 2.1: Examples of laser scan data from Japan and Chile following the earthquakes and tsunamis. (a) Topographic mapping in Onagawa, Japan following the tsunami destruction. (b) Steel frame structure in Yuriage, Japan damaged by tsunami impact. (c) Damaged walls from pressurization at a concrete ware- house in Onagawa, Japan. Tsunami damage to (d) the City Hall and (e) the Capital 100 Hotel in Rikuzentakata, Japan. (f) Deformation analysis of a concrete wall blow-in at the Gamou Wastewater treatment plant. (g) Damage to a concrete deck at a port facility in Talcahuano, Chile.[11] 


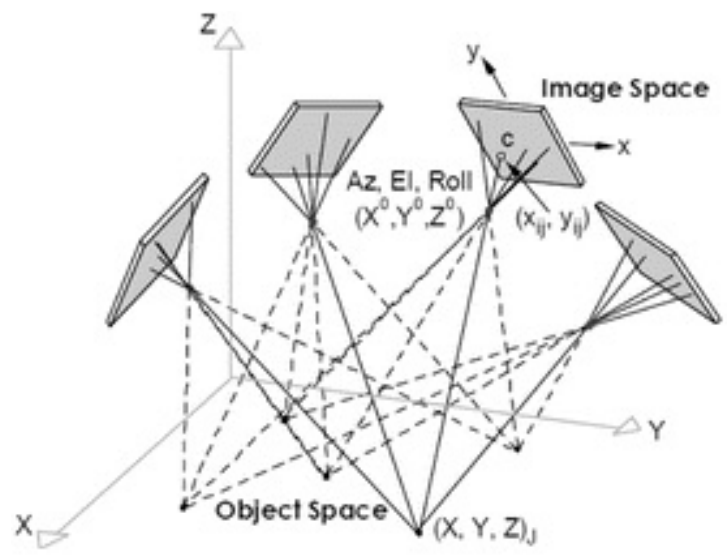

(a)

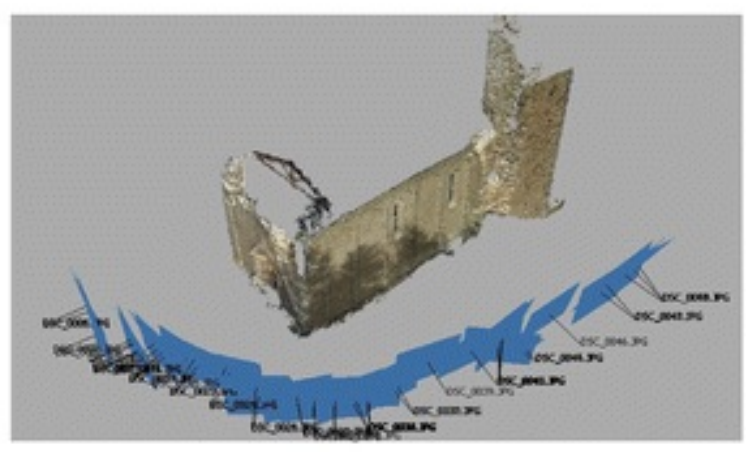

(b)

Figure 2.2: (a) Multi-image configuration. (b) Cameras geometry configuration for in-field acquisition [5]

Behrouzi's project. While there is no mention of having non-experts taking these photos, there is no foreseeable reason why civilians could not provide a majority of the data. Since their work focuses on reconstructing 3D models from 2D images their project is completely different than ours, other than the aim is to help in reconnaissance efforts. Figure 2.2 shows how the building in this study was recreated digitally using photos of the building.

\subsubsection{Visual Data Classification in Post-Event Building Reconnaissance}

This work is closely related to the work that this thesis is a part of. The project's goal is to automatically classify images taken after an earthquake. The authors of this Visual Data Classification project are aware of the inconsistency of the expert taggers but the solution in this project is to make sure all images are tagged by three different people. Since there were many inconsistencies on the images the authors concentrated on just recognizing one damage type: Spalling, since this is one of the damage types that is easiest to recognize and tag consistently. The authors use a similar way of training their artificial intelligence by using a set of ground truth marked images [17]. Figure 2.3 shows an example of some of the images taken for 



Figure 2.3: Samples of ground truth (labeled) images: (left) collapse and (right) spalling [17].

this project and the "ground truth" tags that have been made on them. In the study, the authors implemented the popular ImageNet convolutional neural network called Axelnet to tag images of spalling damage.

Unfortunately spalling is a minor damage and mostly cosmetic. In the project being worked on by Dr.Pantoja and Dr. Behrouzi, the goal is to tag all of the different damages and to quantify how inconsistent the damage is tagged by the expert. 
Chapter 3

\section{SYSTEM DESIGN}

This chapter describes the system overview, including how the work in this thesis fits into the larger project as well as some of the design decisions that were made during development.

\subsection{System Overview}

This section briefly describes the overall system of which this thesis is a part of. See Figure 3.1 for the system's execution flow.

The input data to the project is a set of about 200 untagged images. These images are presented to either an expert or a trainee in a slide show manner for tagging purposes. Experts and trainees alike utilize a graphical user interface (GUI), described bellow, to mark the images with rectangles and to select damage tags. The output of the GUI is a text file containing the image and rectangle data. These text files are then used as inputs to the Fast Feedback Tool developed in this thesis. The feedback tool reads the data, conducts an evaluation and outputs scores for each

image in the files as well as a total score. These outputs are either used by trainees to determine how successful they were at marking images or by as training data for a neural network (NN). Full details of the implementation of the feedback tool is provided in Chapter 4. 


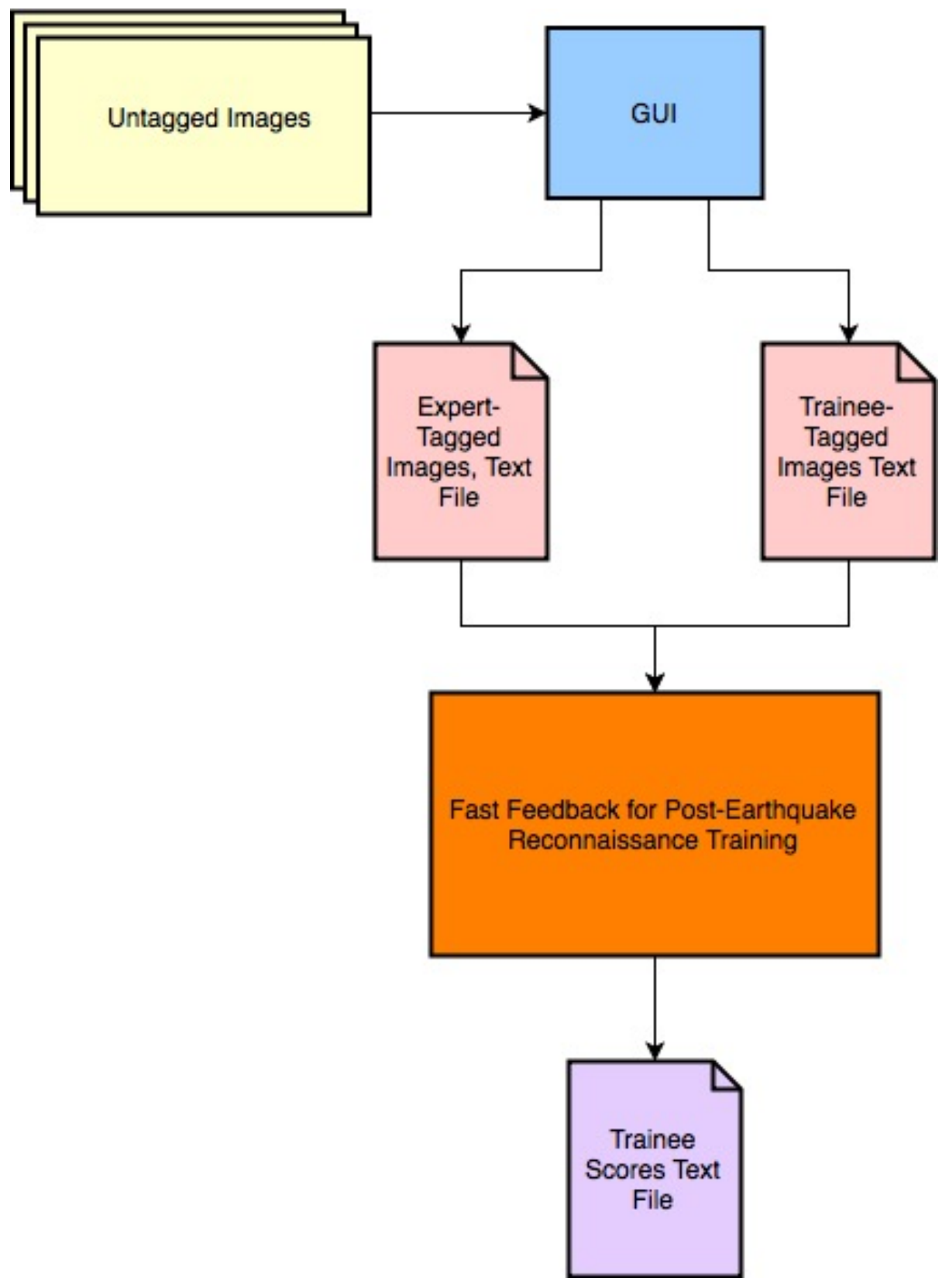

Figure 3.1: System overview diagram 


\subsection{Photo Tagging Tool for Rapid and Detailed Post-Earthquake Struc- tural Damage Identification}

The work of this thesis is built off of the Photo Tagging Tool for Rapid and Detailed Post-Earthquake Structural Damage Identification project [4].

The aim of the project in [4] is to provide a training mechanism to allow for non-experts to learn to take photographs and tag images of buildings affected by earthquakes. Once the training course has been completed, civilian volunteers would be able to travel to areas affected by major disasters to help determine the amount of damage sustained by the buildings in the area. Using a standardized set of tags to mark the images they take, the volunteers would greatly accelerate the process of determining which buildings are safe for use. With the vast number of volunteers interested in contributing to the provision of aid in the affected areas [7], a scalable training solution is needed.

This project uses a training set of images that have been previously tagged by an expert. These images are photographs of buildings damaged in previous earthquakes. Un-tagged images are provided to the trainees in an easy to navigate graphical user interface displaying the photos from the training set in a slide show sequence. Trainees will outline damaged area(s) of the building in the image with rectangle(s) and select an associated damage-structure pair from a set of radio buttons. Figure 3.2 shows what the GUI looks like for users.

The work in [4] outputs text files from an open-source software package, which consists of an easy-to-navigate GUI that displays untagged photographs from the ground truth set in a slide show sequence. The trainee is prompted to draw a bounding box around the damage(s) shown in each image and select an associated damage-structure pair from a set of radio buttons. Upon completion of tagging all photographs in the 


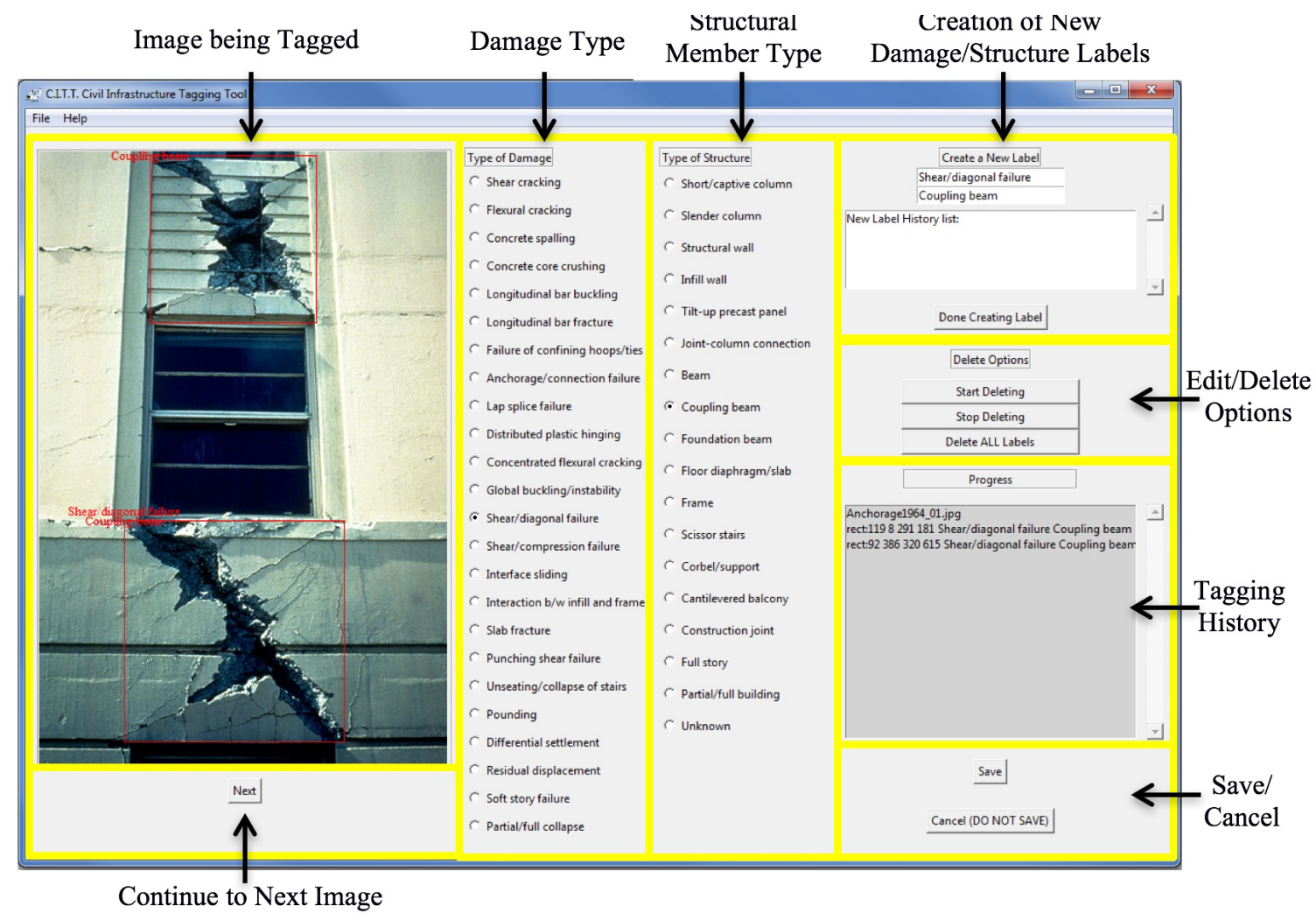

Figure 3.2: Reinforced concrete building image tagging module (yellow boxes and labels added) [4]. 
slide show, the Fast Feedback Tool will promptly provide a formative assessment both by indicating the trainees percent accuracy compared to the expert and specific feedback about the most common errors to indicate their readiness to tag other images or perform reconnaissance tasks. This thesis primarily focuses on providing the percent accuracy comparison in a timely fashion.

The aforementioned problem of evaluating trainee results against the expert is not as simple as comparing and finding differences between two sets of image files. This thesis faces challenges in that each trainee will select a slightly different sized area for the same occurrence of damage, and some damage-structure pairs are more difficult to recognize and tag. Additionally, this tool is intended to be used in conjunction with free earthquake reconnaissance webinars that train responders, similar to the one recently hosted by the Applied Technology Council (ATC) in the aftermath of Hurricane Harvey [4]. Historically, these webinars have a heavy attendance and it will be necessary to compare the ground truth set to files from hundreds of trainees; therefore, the research team has selected to implement the algorithm using parallel paradigms to accelerate the computation time.

The output from the photo tagging tool is a text file representing the locations of the rectangles and their associated damage tags for each image in the test set. Currently, each the text file will represents either a new image name or the rectangles associated with the image. Each line that denotes an image contains the image name. Each line that denotes a rectangle starts with the characters 'rect:' followed by four integers representing the $\mathrm{X}$ coordinates of the left and right edge of the rectangle as well as the $\mathrm{Y}$ coordinates of the top and bottom edge of the rectangle. These coordinates are in the order $\mathrm{x} y \mathrm{x} \mathrm{y}$ but there is no order to which coordinate is top vs bottom or right vs left. After the damage tag is a string representing the damage/structure tag pair that was selected using the radio buttons on the GUI. The work in this thesis does not accept files of this type which is discussed later and a 
change is set to be made on the photo tagging tool such that the output will have enumerated integer values to represent the damage tags instead of strings. How this discrepancy is handled for testing purposes is covered in Chapters 4 and 5. Sample data is provided below:

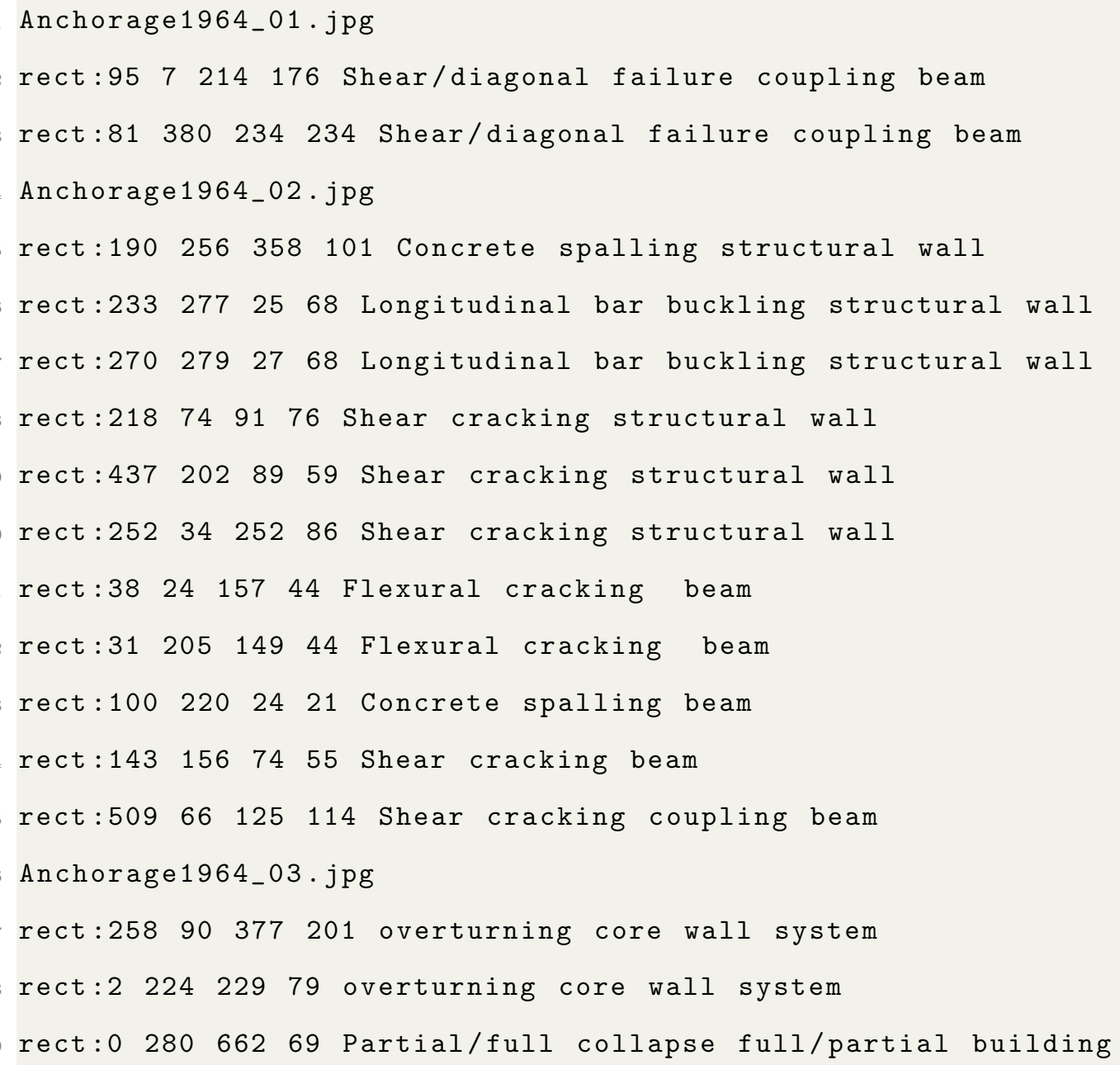

\subsection{Early Approaches}

This section briefly describes some of the early approaches to creating this system that were ultimately not used in the Fast Feedback Tool. 


\subsubsection{Language Decision}

Originally, the project was started in $\mathrm{C}$ and used type defined structs to store the image and rectangle data. C was selected for it's simplicity and its ability to be converted to CUDA fairly easily. The struct for representing an image contained a preallocated array for the image name as well as a preallocated array of rectangle structs. The rectangle struct held values for the coordinates of each rectangle edge and a preallocated array for the damage tag name. Image structs were held in a preallocated array and filled in when the image files were read in. All of these preallocated arrays caused space problems, and the need for dynamic allocation of data space was a primary reason for the move away from C. It was also in this part of the project that strings for tag values were changed to integers to save space and time while reading image data and comparing the images.

\subsubsection{Rectangle Comparison}

The first rectangle comparison method used in this tool was a three step comparison process that utilized the dimensions of the image to determine if two rectangles were a match.

\section{Tag Comparison}

Checked if the tags were the same. If they were, then the rectangle positions and sizes would be checked.

\section{Rectangle Center Comparison}

Using the image dimensions, the two centers of the rectangles were compared to determine if the centers of the two images are close together. If the difference between the coordinates of the centers of the rectangles in both the $\mathrm{X}$ and $\mathrm{Y}$ direction were within a small percentage of the image dimension in that 
direction, then the rectangles were a potential match. The use of the image dimensions made it so that if the image was large and higher dimension, then the trainee would be allowed to be off by more pixels. In the case of a higher resolution image, it would be more difficult to match the rectangles perfectly with the instructor's, and the use of the image dimensions accounts for this.

\section{Rectangle Area Comparison}

If the centers were close enough, then the rectangle areas were compared. This ensured that even if the centers were close together, if the trainee had boxed a much larger or much smaller space, then the rectangles would not count as a match. Again, the image's dimensions were used to calculate how much the trainee could be off by, giving more room for error on higher resolution images. The percentage used to determine how much the rectangles' areas could be different by was much smaller than the percentage used to compare the centers, since the areas of the rectangles would end up being much much greater than the differences between the coordinates.

If a match for a rectangle was found, then it was counted as a perfect score for that rectangle. If a second match for the same rectangle was found then the two matches had to be compared to determine which was better. This comparison was somewhat complicated, as a weight had to be assigned to how close together the centers were versus how close the areas were to each other.

Ultimately, this comparison method was abandoned for a better one, the Jaccard Index [14] described bellow in Section 3.5. The primary reasons for the switch were that the original method was overly subjective, in that the percentages of image dimensions were fixed and arbitrarily chosen. Additionally, this method could only

give a whole value score for matching rectangles, while a fractional value that could describe how close the trainee's rectangle was matching is more desirable. 


\section{$3.4 \mathrm{C}++$}

After determining that $\mathrm{C}$ would not be a suitable language for this project, $\mathrm{C}++$ was selected. Primarily, $\mathrm{C}++$ provides much easier dynamic allocation of data than $\mathrm{C}$, while still being easily converted to CUDA, an end goal of the project that this thesis is a part of. Furthermore, $\mathrm{C}++$ provides object oriented programming that $\mathrm{C}$ does not, and the data storage needs for rectangles and images lend themselves nicely to being implemented as classes. $\mathrm{C}++$ is also a language that I felt comfortable with using for this kind of project.

\subsection{Jaccard's Index}

This section describes Jaccard's index of similarity and its application as a method for comparing rectangles in this thesis. Jaccard's index is a statistical method for determining the degree to which two things are similar [14].

Jaccard's index was originally developed by Paul Jaccard, a professor of botany and plant physiology in the early 1900's. Jaccard's index is a statistic used for determining the degree of similarity between two finite data sets. While originally developed for classification of flora, its usefulness has branched into other disciplines such as ecology, biogeography, and even computer science. The index can be used to compare two operational taxonomic units [14]. An operational taxonomic unit is a term used to describe two closely related individuals. The term OTU was originally

coined to describe the groups of organisms being studied [16]. Jaccard's index is a good technique for this type of comparison because the index is not affected by OTU's that are not being compared [14].

Jaccard's Index can be represented mathematically in a couple different ways. The most simple and common representation is the intersection of two data sets over 


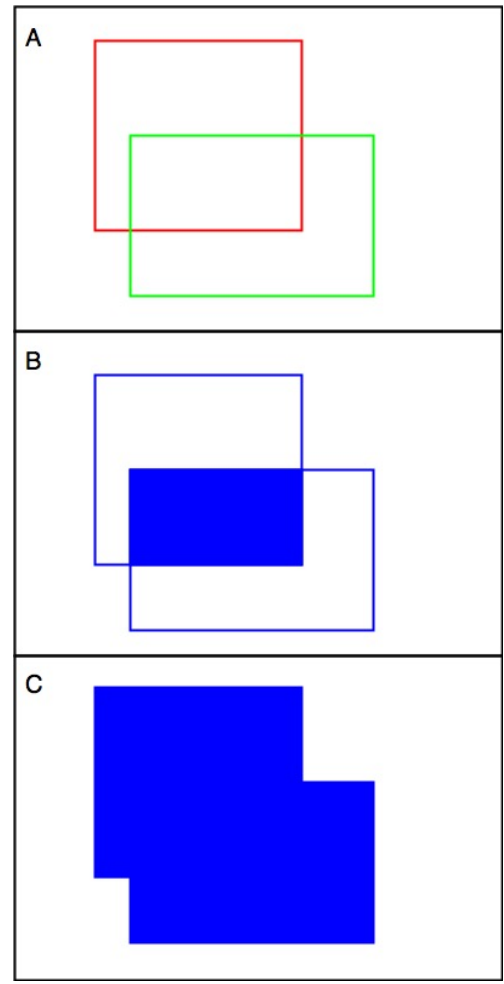

Figure 3.3: Example of Jaccard's index applied to rectangle areas

the union of the two data sets.

$$
\frac{A \cap B}{A \cup B}
$$

This can also be written as

$$
\frac{C}{A+B-C}
$$

Where $\mathrm{A}$ is the attributes present in OTU a, B is the attributes present in OTU $\mathrm{b}$, and $\mathrm{C}$ is the elements present in both OTUs a and b [14].

Jaccard's index can be applied to the work in this thesis as a method for comparing two rectangles to determine if they are a match meriting a score. This can be done by using the areas of the rectangles. 
Jaccard's index for rectangle comparison is calculated using Equation 3.2, where $\mathrm{A}$ is the area of rectangle a, B is the area of rectangle $\mathrm{b}$, and $\mathrm{C}$ is the area of overlap between rectangles a and b. Figure 3.3 shows an example of two rectangles being compared, the area of overlap, and the area of the union. One of the advantages of using Jaccard's index for comparing two rectangles is that it provides a fractional value for how closely the rectangles are matching. See the Implementation Chapter Section 4.2.2 for an example of Jaccard's index being applied to the input data. This fractional score is a more desirable outcome from comparison as it provides the trainees with a better idea of how accurate their tags have been. However, Jaccard's index does have its draw backs. Due to the way Jaccard's index is calculated, scores decrease dramatically as the overlap between the two sets decreases. This is covered in further detail in Section 5.3.3. 
Chapter 4

\section{IMPLEMENTATION}

This chapter describes the details of the implementation of the Fast Feedback Tool.

\subsection{Class Models}

This section describes the classes created to solve this problem. Two main classes were made to handle data used in this program. The first is an Image Class and the second is a Rectangle Class. For clarity purposes Image and Rectangle with uppercase I's and R's will represent Image/Rectangle classes or objects while image and rectangle with lowercase i's and r's will represent the images used as input to the GUI or the rectangles that were drawn on the images.

\subsubsection{Image Class}

The Image Class is used to hold data for a single image from the training set. The .cpp and .hpp files for the Image Class can be found in the appendix. An overview of the data that is held in the Image class is given here.

- Name The name of the image file. (string)

- Number of Rectangles (int) The number of rectangles that were drawn on the image by the person who has tagged the image.

- Total Score (double) The total score for the image, in the form of some decimal value between zero and the number of rectangles. Used as a measurement for how accurate the trainee was when tagging the image. 


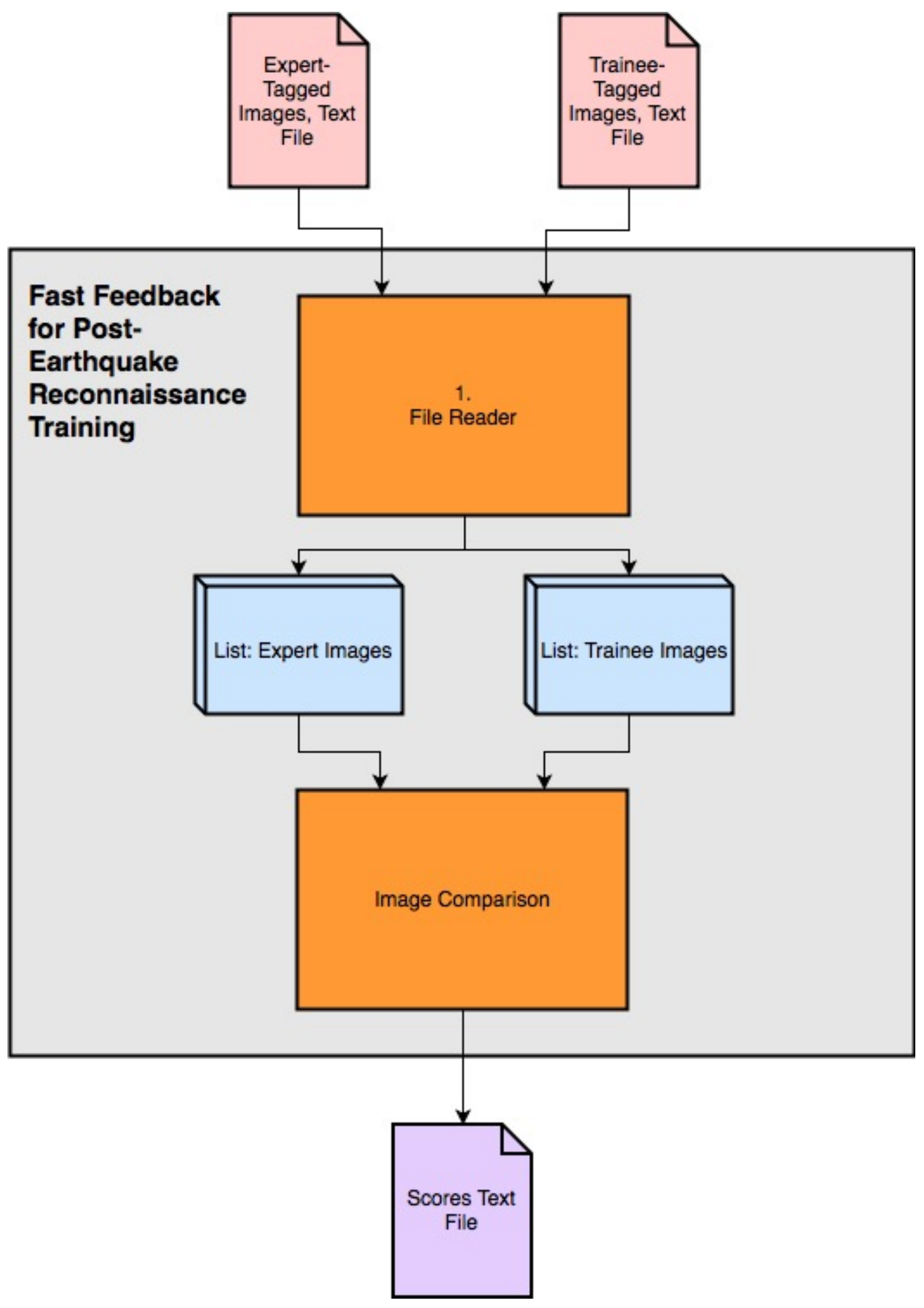

Figure 4.1: Flow diagram for implementation of the Fast Feedback Tool 
- Rectangle List (List: Rectangles) List of Rectangle Objects representing the areas tagged on the image.

- Number of Images (int) Class wide attribute that keeps track of how many Image Objects have been created.

\subsubsection{Rectangle Class}

The Rectangle Class is used to hold data for a single rectangle marked on an image. The .cpp and .hpp files for the Rectangle Class can be found in the appendix. An overview of the data that is held in the Rectangle Class is given here.

- X coordinate (int)(2) the horizontal position of the vertical edges of the rectangle.

- Y coordinate (int)(2) the vertical position of the horizontal edges of the rectangle.

- Tag (int) An enumeration value representing what damage tag the rectangle has.

- Match (pointer to Rectangle object) The Rectangle Object that matches with this rectangle.

- Match Score (double) The score for the current match representing how closely the two rectangles are matching. This is a value between 0 and 1 .

- Number of Rectangles (int) Class wide attribute that keeps track of how many Rectangle Objects have been created. 


\subsection{System Details}

The problem of providing fast feedback to trainees using the image tagging tool described in Section 3.2 proved to be more complicated than simply comparing and finding differences between two sets of image files. There are several subtle problems that arise from the way that images are tagged and output is generated. Since rectangles are being marked on images, it is highly unlikely that a trainee will line up the rectangle perfectly with the area marked by the expert. Depending on the resolution of the image, the rectangles may be misaligned by many pixels. Furthermore, the order in which the trainee marks rectangles on the image is the order in which the rectangles will appear in the text file output used to compare the trainee's work to the expert's. The expert may have marked the rectangles in a different order. In order to match the rectangles from the trainees' solutions to that of the experts, each rectangle must be compared against all other rectangles. Following is a detailed description of how the solution to these problems is implemented.

\subsubsection{Reading Input Data}

The input to this program is the text file output by the image tagging tool. The text file consists of image and rectangle data. Each line denotes either a new image name or the rectangle data for the most recent image. Rectangle data consists of a line of text starting with the characters 'rect:' followed by five distinct integer values. In order, the integer values are: $\mathrm{X}$ coordinate for the first vertical edge, $\mathrm{Y}$ coordinate for the first horizontal edge, $\mathrm{X}$ coordinate for the second vertical edge, Y coordinate for the second horizontal edge, and an enumeration value for the type of damage that the rectangle is tagging. The coordinates will always be in $\mathrm{X}-\mathrm{Y}-\mathrm{X}-\mathrm{Y}$ order but the order of the $\mathrm{X}$ coordinates and the order of the $\mathrm{Y}$ coordinates depend on the way in which the person tagging the image drew the rectangle. For example, if the user drew a 


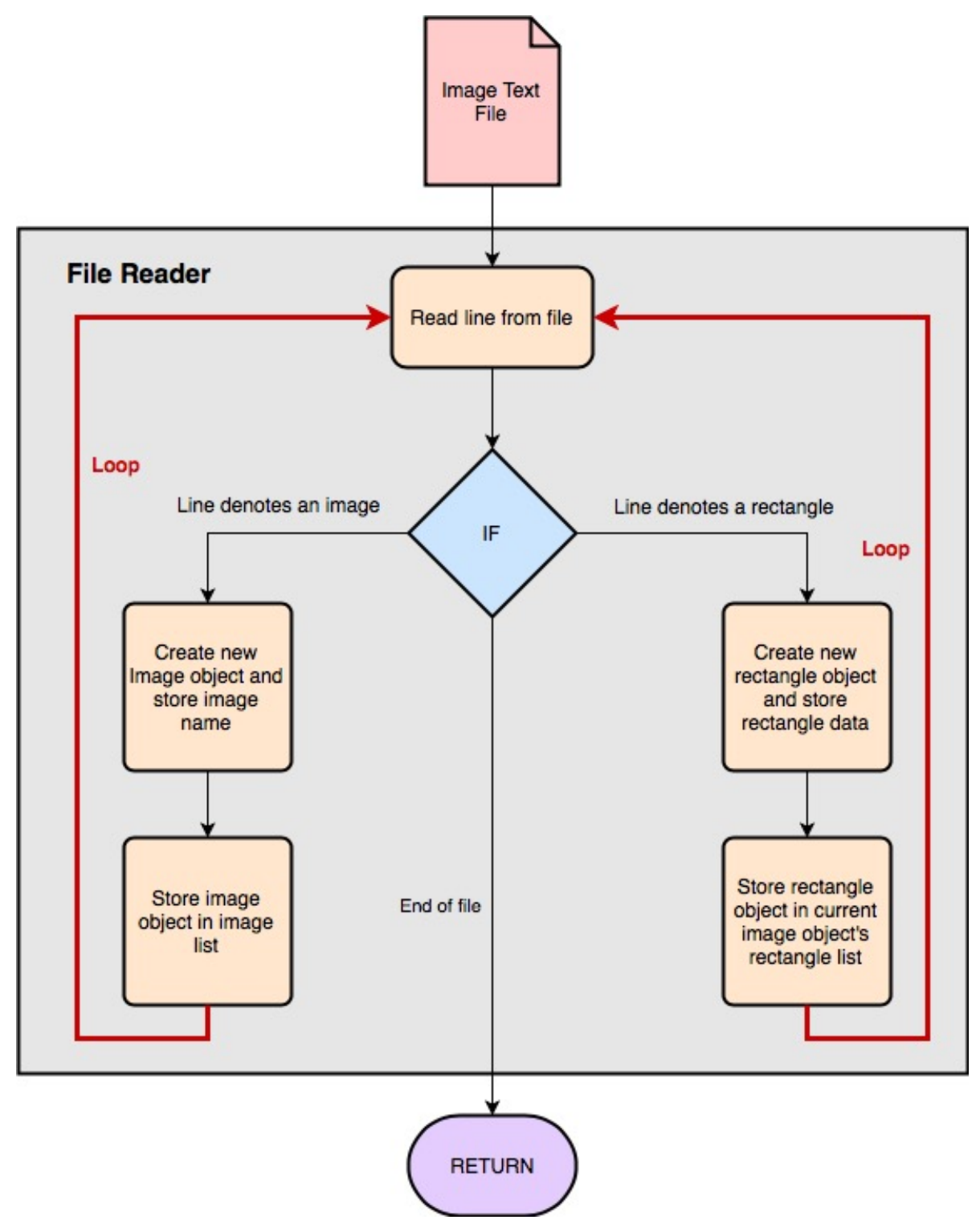

Figure 4.2: Flow diagram for the getImgData function 
rectangle by clicking in the top right and dragging to to bottom left, the $\mathrm{x}$ coordinate for the right side of the rectangle would be first, followed by the $\mathrm{Y}$ coordinate for the top of the rectangle, then $\mathrm{X}$ coordinate for the left of the rectangle and lastly the $\mathrm{Y}$ coordinate for the bottom of the rectangle. Creating the same rectangle by starting in a different corner would result in the order of the coordinates being different while maintaining the X-Y-X-Y coordinate order.

The file reader function ignores these rectangle and coordinate ordering nuances and simply stores the data for the images and rectangles in whatever order they appear in the input file. Figure 4.2 shows program flow for the file reader function. The file reader takes two inputs. The first is the file path to the text file that will be read. The second is the $\mathrm{C}++$ list used to store addresses of Image Objects. The function has no return values and fills the list through side effect.

For each line in the input file, the file reader checks if the line denotes a rectangle or an image.

1. If the line denotes an image, the function creates a new Image Object, stores the image's name in it and stores the Image Object's address in the $\mathrm{C}++$ list.

2. If the line denotes a rectangle, the function creates a new Rectangle Object, and stores the rectangle's coordinates and tag data in the Object. The Rectangle Object's address is then stored in the current Image Object's rectangle list.

3. If the end of file is reached, the function returns.

Once the file reader function returns, the Image list that was input should be filled with addresses to Image Objects which are in turn filled with a list of addresses to the Rectangle Objects which store the rectangle data for all rectangles that were marked on the individual images. 


\subsubsection{Image Comparison}

The portion of the system responsible for analyzing image data and calculating the comparison scores is broken down into several subsystems for simplicity and code modularity. Additionally, there are four versions of comparison, one is a fully serial version of the program and the other three are different parallel versions, created to determine the best way to accelerate feedback for the trainees. The details of these different implementations is described in this section.

\section{Serial Comparison}

The serial version of image comparison is broken down as follows:

\section{1. imageCompareSerial}

The imageCompareSerial function has two inputs, a list of pointers to Images from the expert's solutions and a list of pointers to Images from the trainee's solutions. Figure 4.3 shows program flow for this function. A for loop is used to select each Image Object in both the trainee and expert list of Image Objects. The pairs of Images are used as inputs to the rectangleCompareSerial function to compare the rectangles in each image. The imageCompareSerial function has no return value and the values of the Image object scores are changed through side effect.

\section{2. rectangleCompareSerial}

The rectangleCompareSerial function has two inputs, a pointer to the expert's Image Object and a pointer to the trainee's Image Object. Figure 4.4 shows the program flow for this function. A for loop is used to select each Rectangle in the expert's Image Object. This Rectangle Object and the trainee Image Object are used as inputs to the traineeRectangleCompare function. Once 


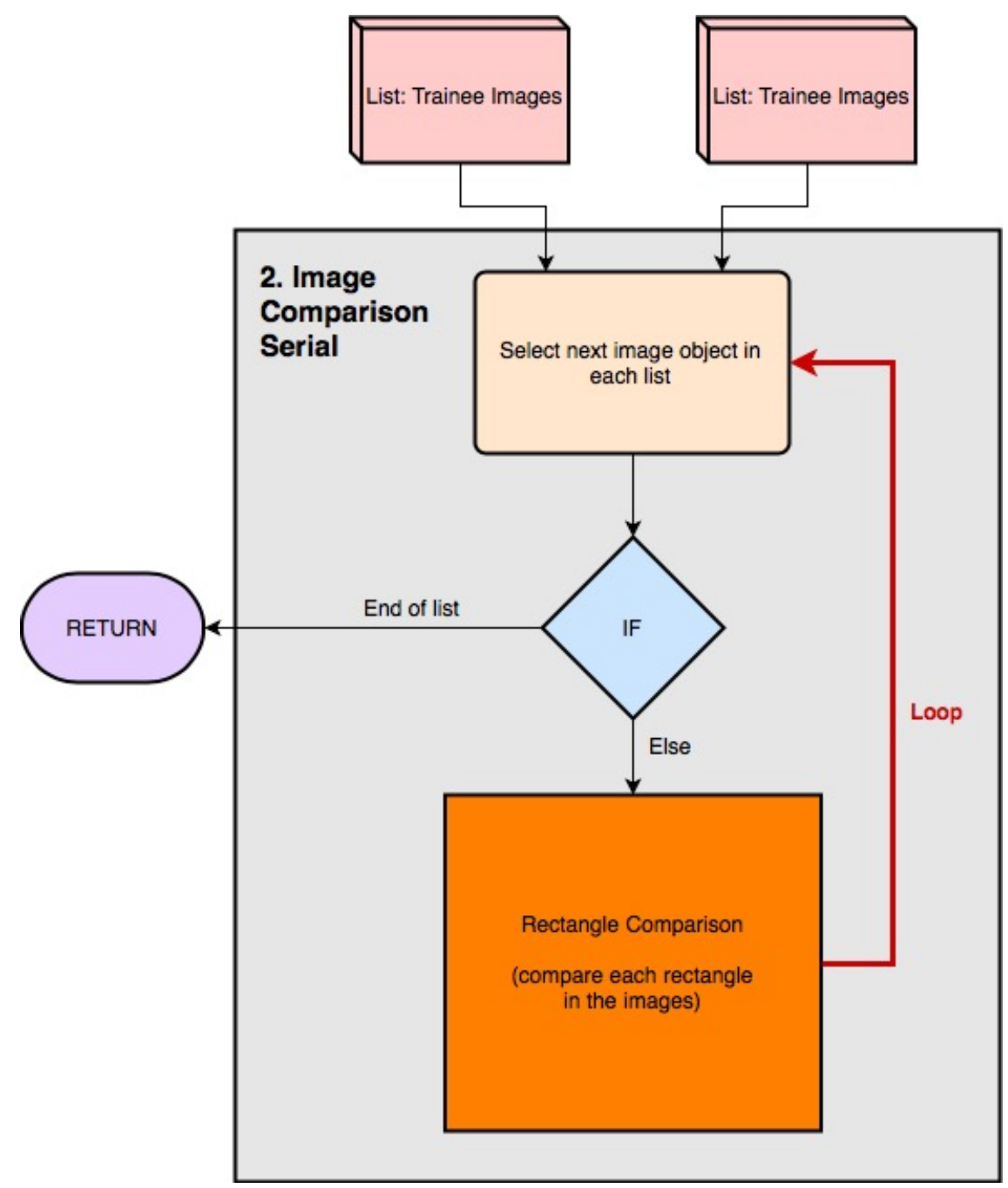

Figure 4.3: Flow diagram for imageCompSerial function 


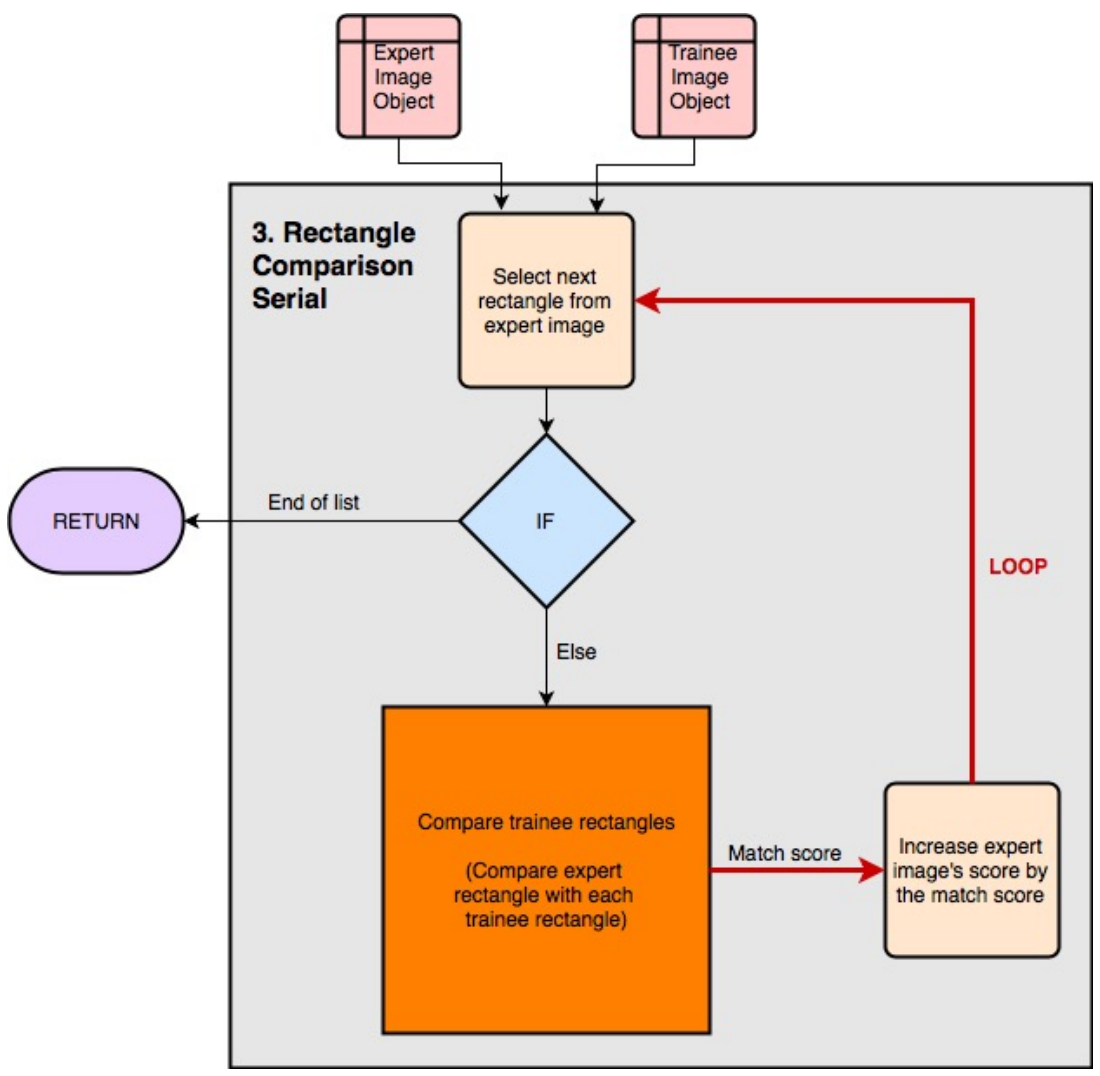

Figure 4.4: Flow diagram for rectangleCompSerial function 


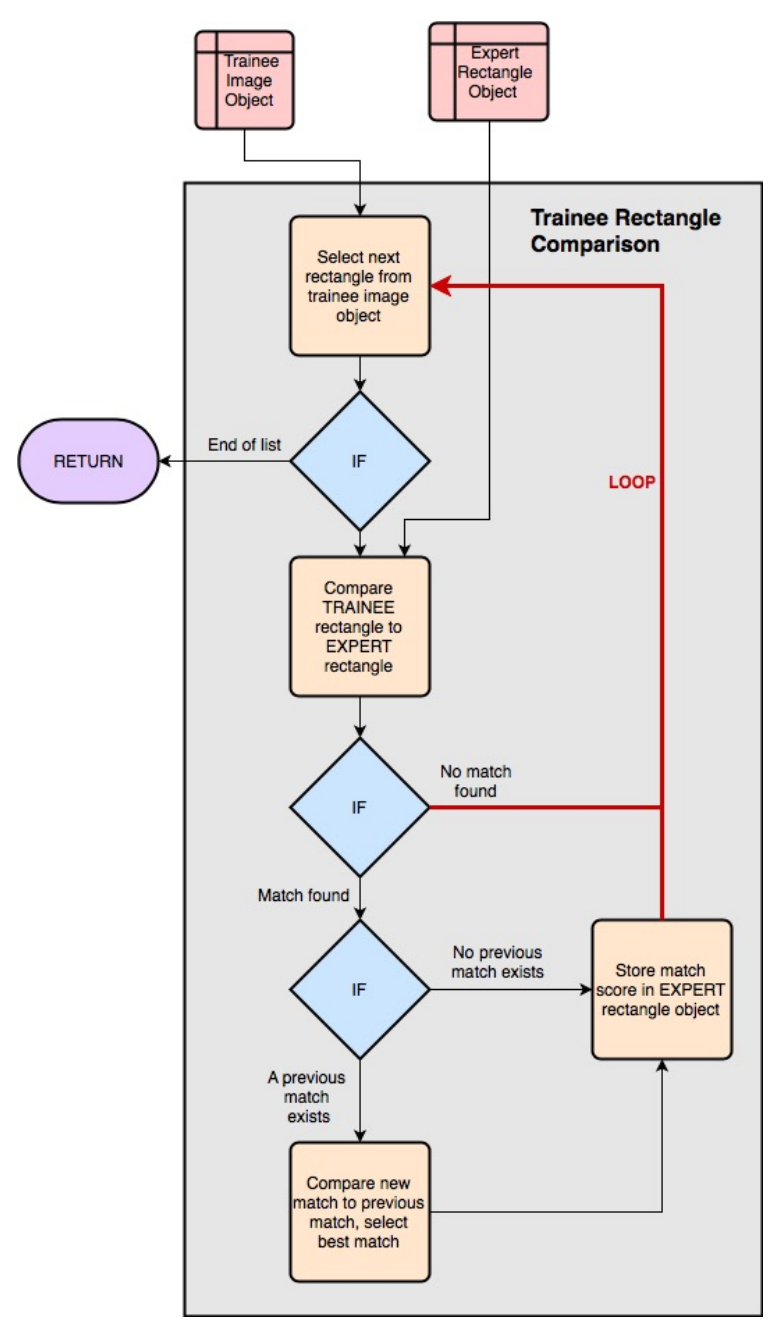

\section{Figure 4.5: Flow diagram for traineeRectComp function}

the traineeRectangleCompare function returns, the expert's Rectangle Object which was used as input to the traineeRectangleCompare function will now hold a score between 0 and 1 which corresponds to the match score calculated in traineeRectangleCompare. This score is then added to the total score for the expert's Image Object. The rectangleCompareSerial has no return value and the expert Image's score is changed through side effect.

3. traineeRectangleCompare

The traineeRectangleCompare function has two inputs: a pointer to the expert's Rectangle Object and a pointer to the trainee's Image Object. Figure 4.5 shows 
the program flow for this function. A for loop is used to select Rectangles from the trainee's Image Object. Each rectangle in the trainee's Image Object is then compared to the expert's Rectangle to find the best matching rectangle. Comparison is done using Jaccard's index, described in further detail in Section 3.5 .

In order to calculate Jaccard's index, the overlapping area of the two rectangles and the total area of the two rectangles must be calculated. Since the edges of the rectangles can come in any order, the rectangle representing the intersection must be found by checking which of the two rectangles top edge is lower, which of the left edges is further to the right, which of the right edges is further to the right, and which of the bottom edges is higher (see Figure 4.6). This will give edges for a potential intersection rectangle. However, if there is no overlap between the two rectangles being compared, then the left edge of the potential intersection area will be to the right of the right edge OR the top edge will be below the bottom edge, resulting in no match. For an example, see Figure 4.6D.

Examples of Jaccard's index being calculated for both intersecting and non intersecting rectangles are given below. The comparison may result in a match being found, and Jaccard's index will provide that match with a decimal score of between 0 and 1 .

Below are two rectangles that overlap a little bit but are not very close, so the score is low.

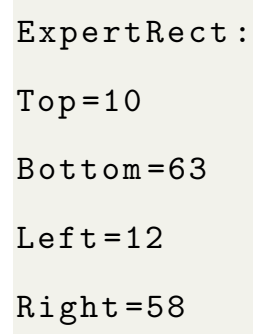




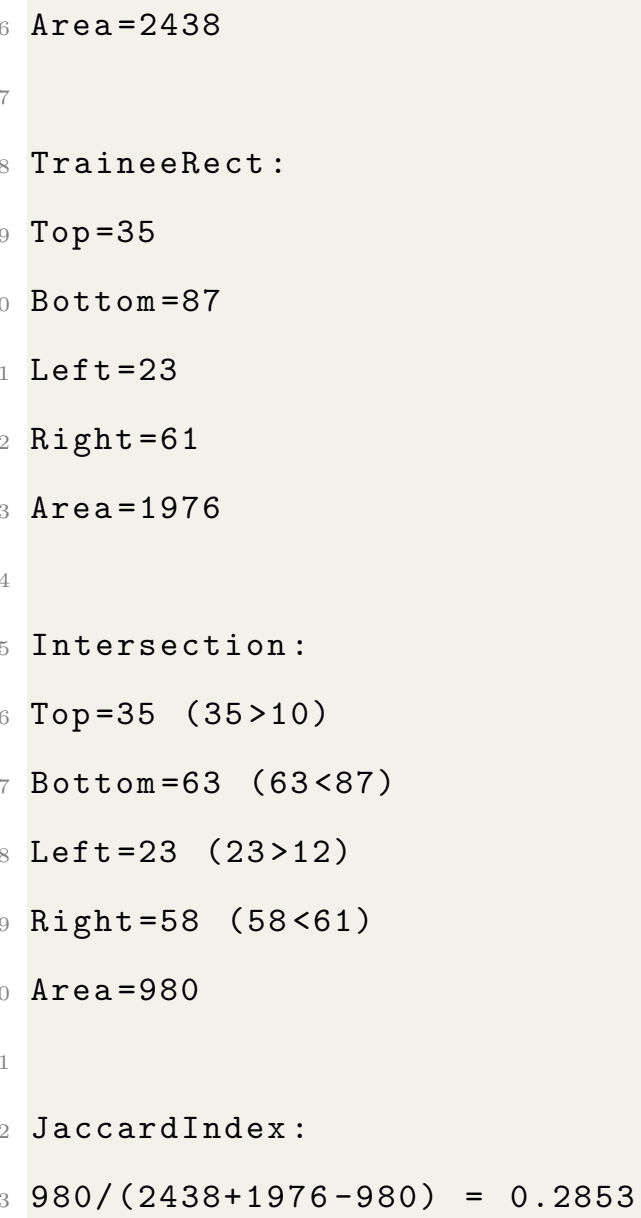

Below are two rectangles that do not overlap since the top of the second rectangle is below the bottom of the second rectangle, meaning that one rectangle is below the other.

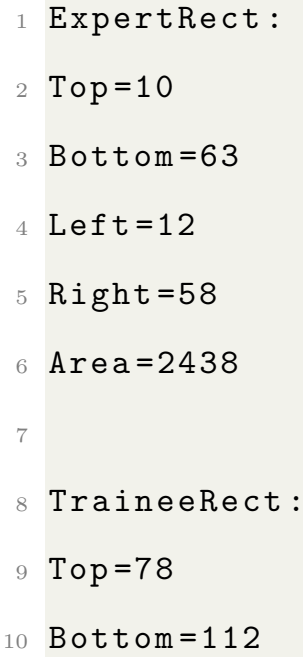




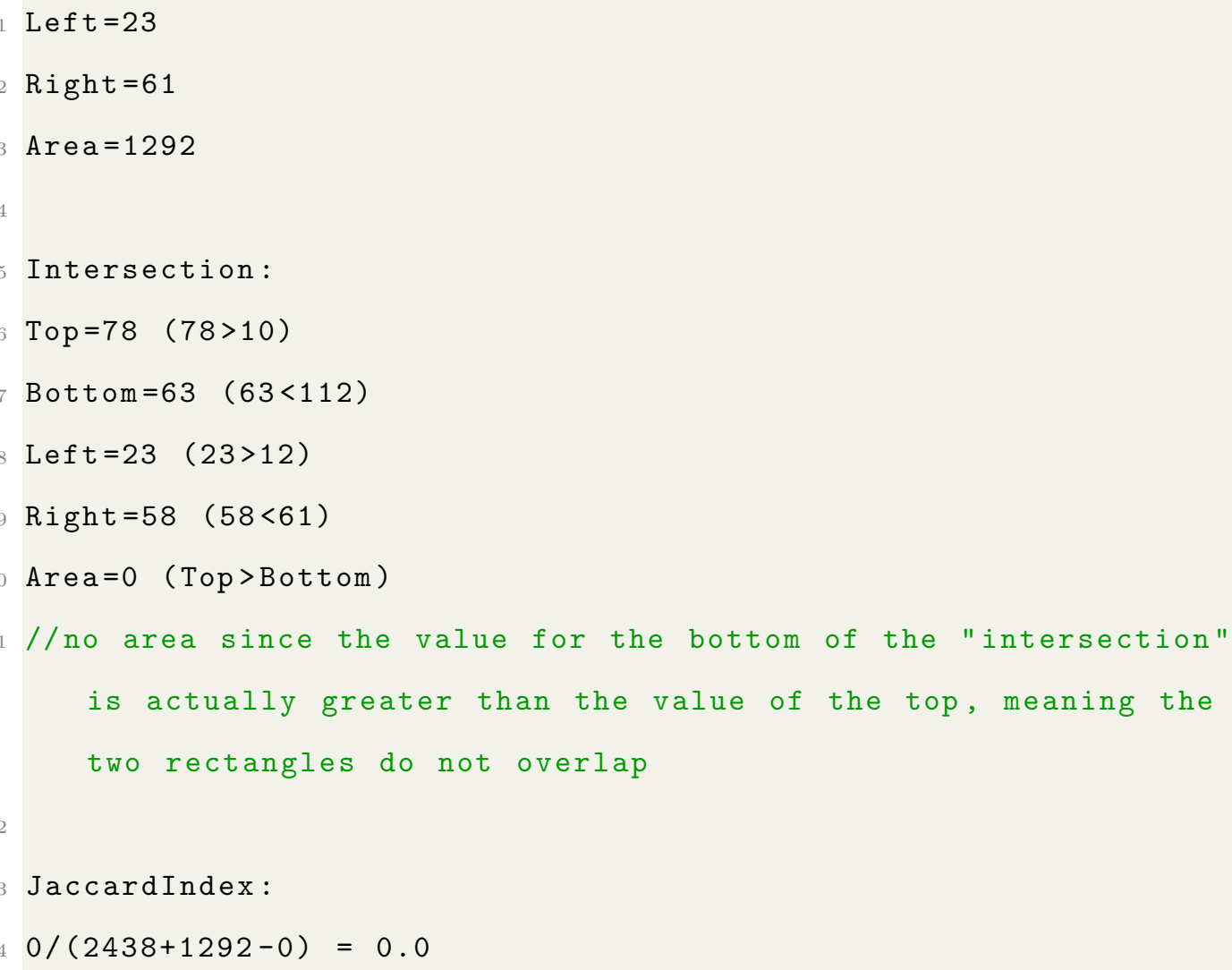

If a match is found and there is no previous match, then the match score is stored in the expert's Rectangle Object. If a match is found and there is a previous match, then the two match scores are compared and the better score is stored in the expert's Rectangle Object. If no match is found, simply check the next rectangle in the trainee's Image Object. The code used to do the comparison between two rectangles is given bellow.

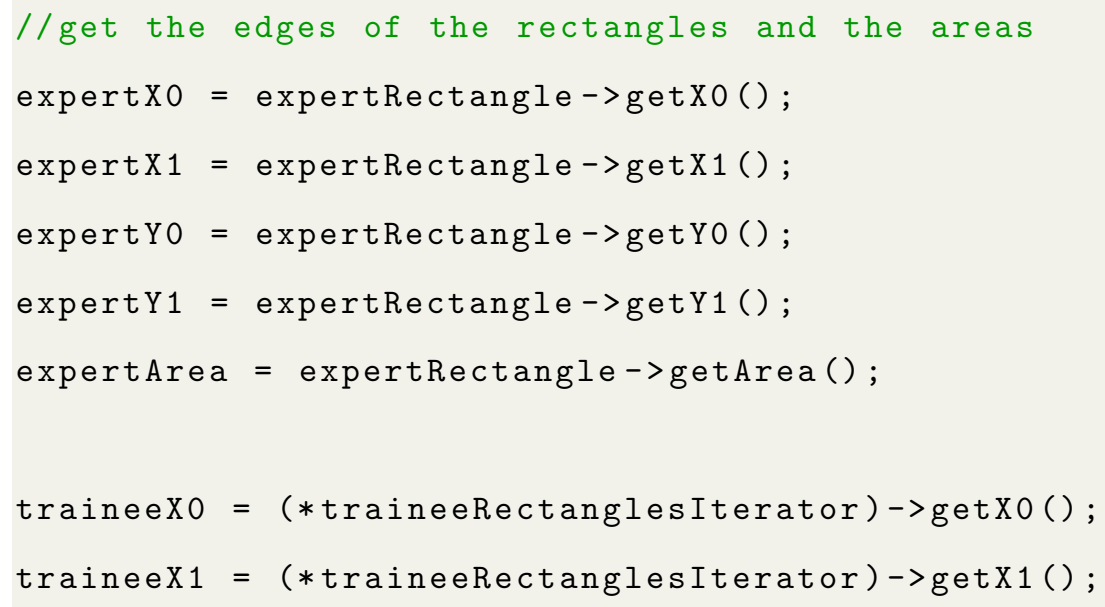




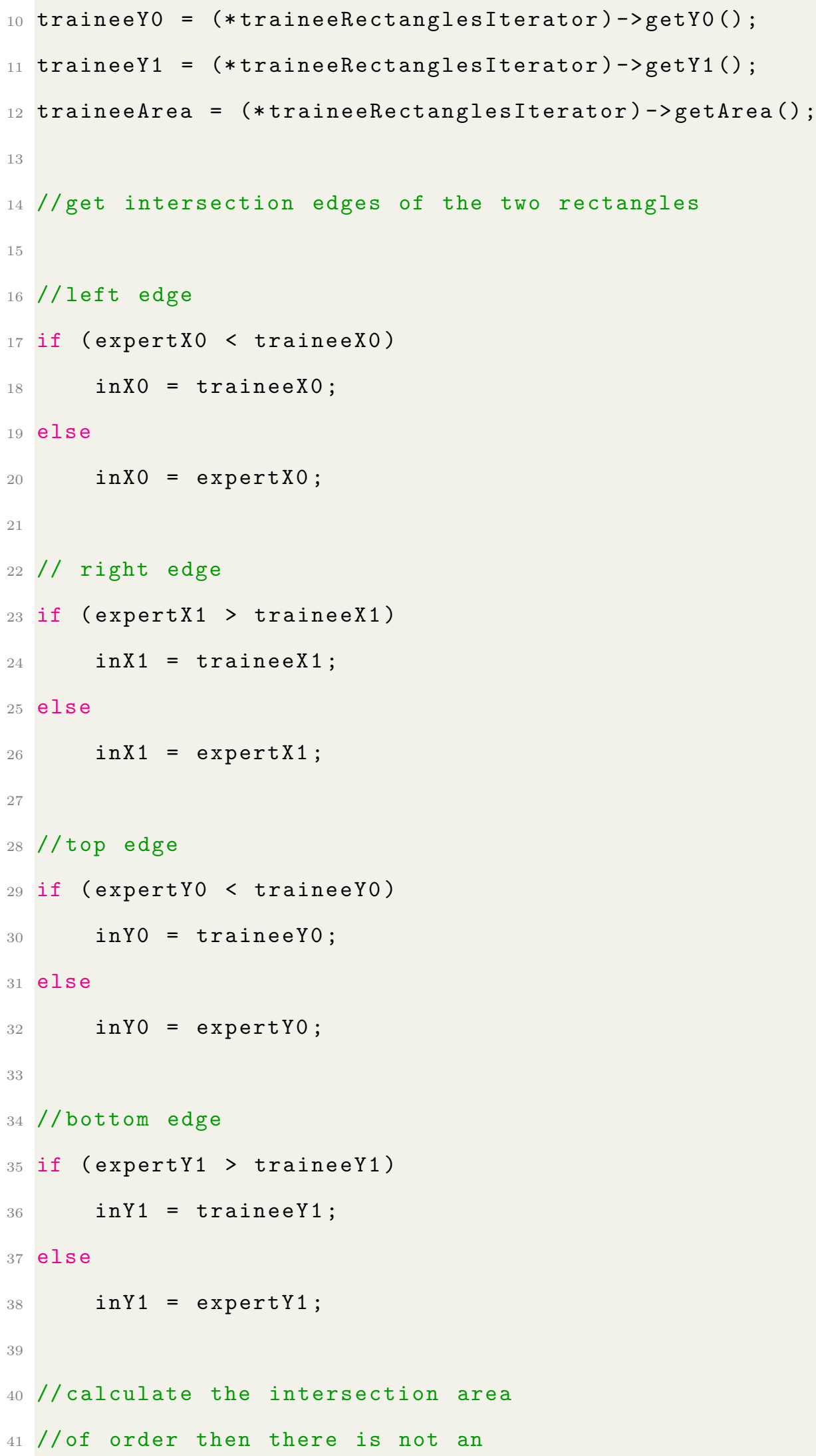




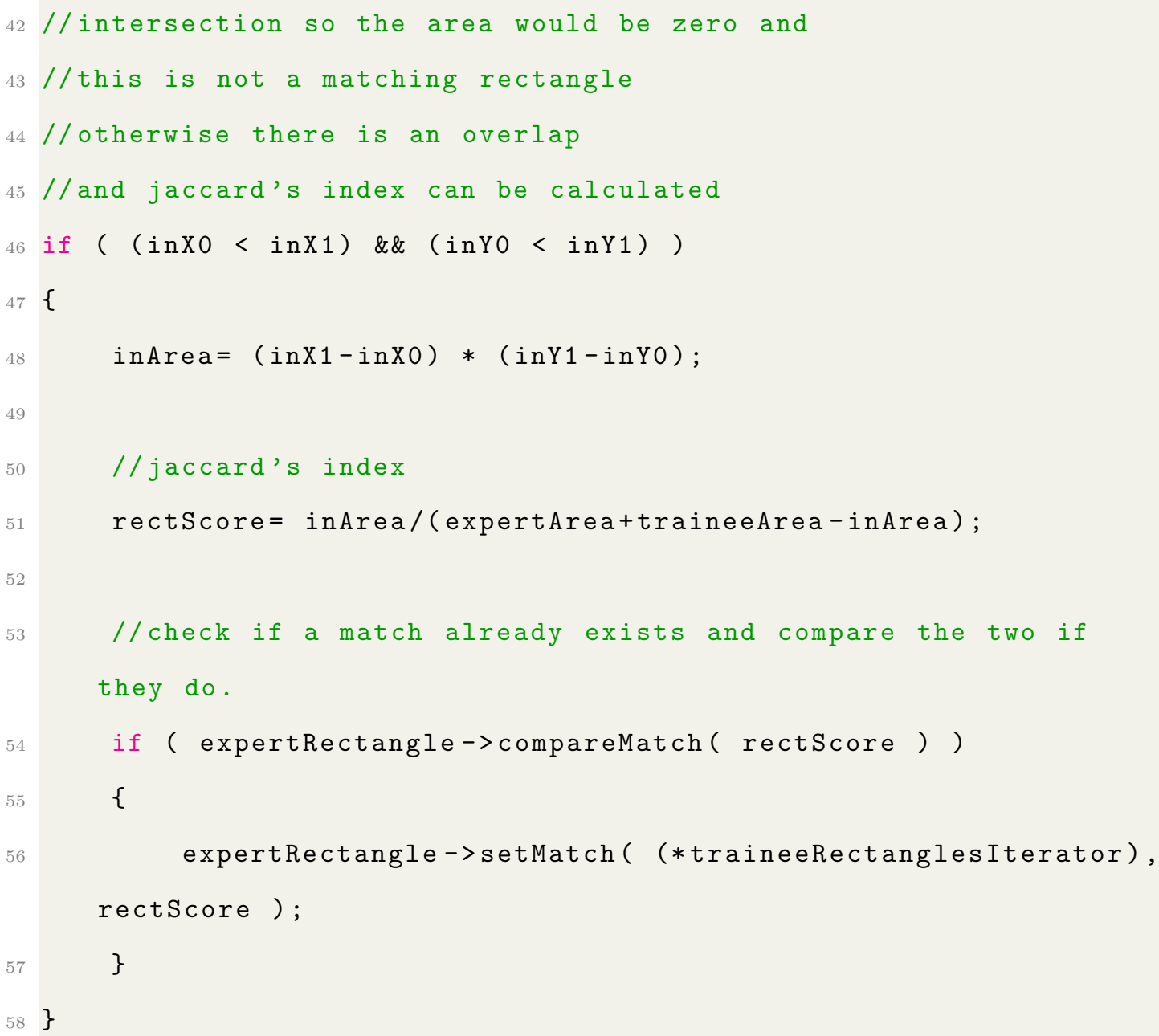

The traineeRectangleCompare function has no return value, and it changes the expert's Rectangle match score through side effect.

Once the imageCompareSerial function returns, the expert Images list should contain scores for each image which can then be output. The scores will be decimal values ranging from 0 to the number of rectangles in the image. These values are then output into a text file for the trainee to view.

\section{Parallel Image Comparison}

The first parallel comparison method targets the image-to-image comparisons for parallelization. This is a good method for parallelization when only a few cores are 


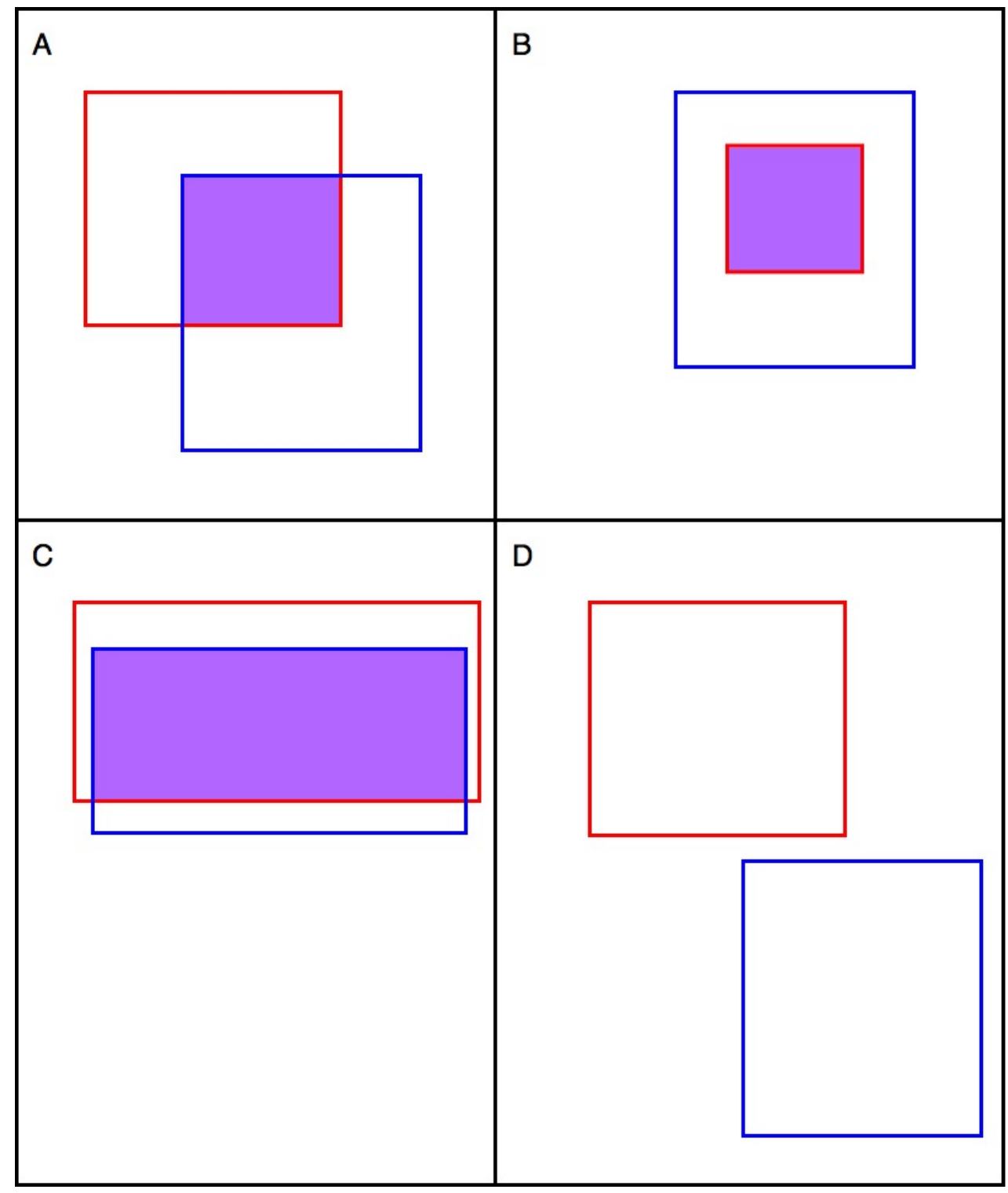

Figure 4.6: Different ways in which rectangles may intersect: A. Top and left edge of intersection from blue rectangle right and bottom edge from red rectangle; B. All intersection edges from same rectangle; C. Three edges from one rectangle; D. No intersection area 
to be used as a large amount of work can be split across a few threads. The details of this method are described here.

1. imageCompareParallelWrapper

The imageCompareParallelWrapper function is the function that actually spawns the threads. See Figure 4.7 to see how this fits with the program flow for parallel image-to-image comparisons. This function has three inputs: a $\mathrm{C}++$ vector of expert Image Object addresses, a $\mathrm{C}++$ vector of trainee Image Object addresses, and the number of threads to be spawned. This function uses a for loop to spawn $\mathrm{C}++$ threads, each running the imageCompareParallel function with the following inputs: both vectors of Image Object, the number of threads to be spawned, and the thread ID. The threads are stored in a vector so that the function can also join the threads after they have finished execution.

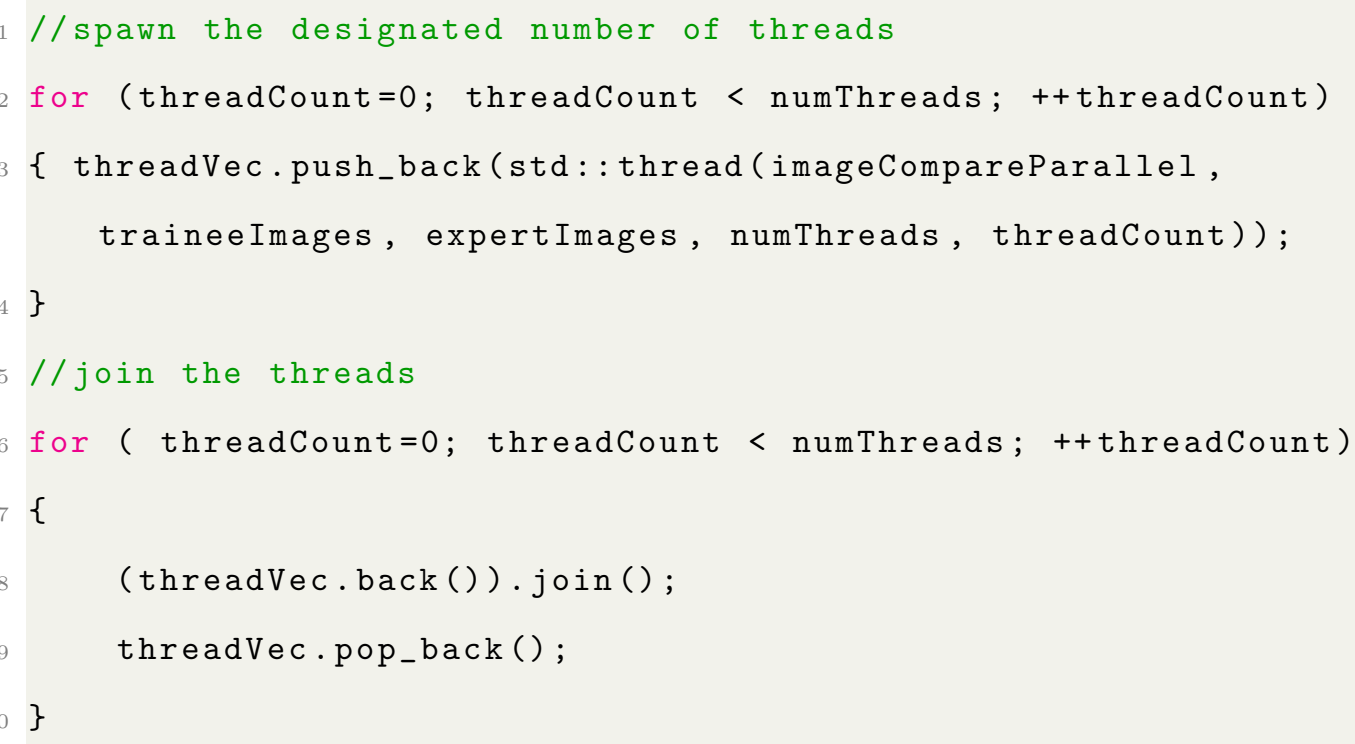

This parallel version uses $\mathrm{C}++$ vectors instead of lists because vectors allow for access to positions in the middle of the data structure. This is important because breaking the images to be compared into blocks to be run on different threads requires that images be selected from the middle of the vector. This would be impossible if a list were being used. 


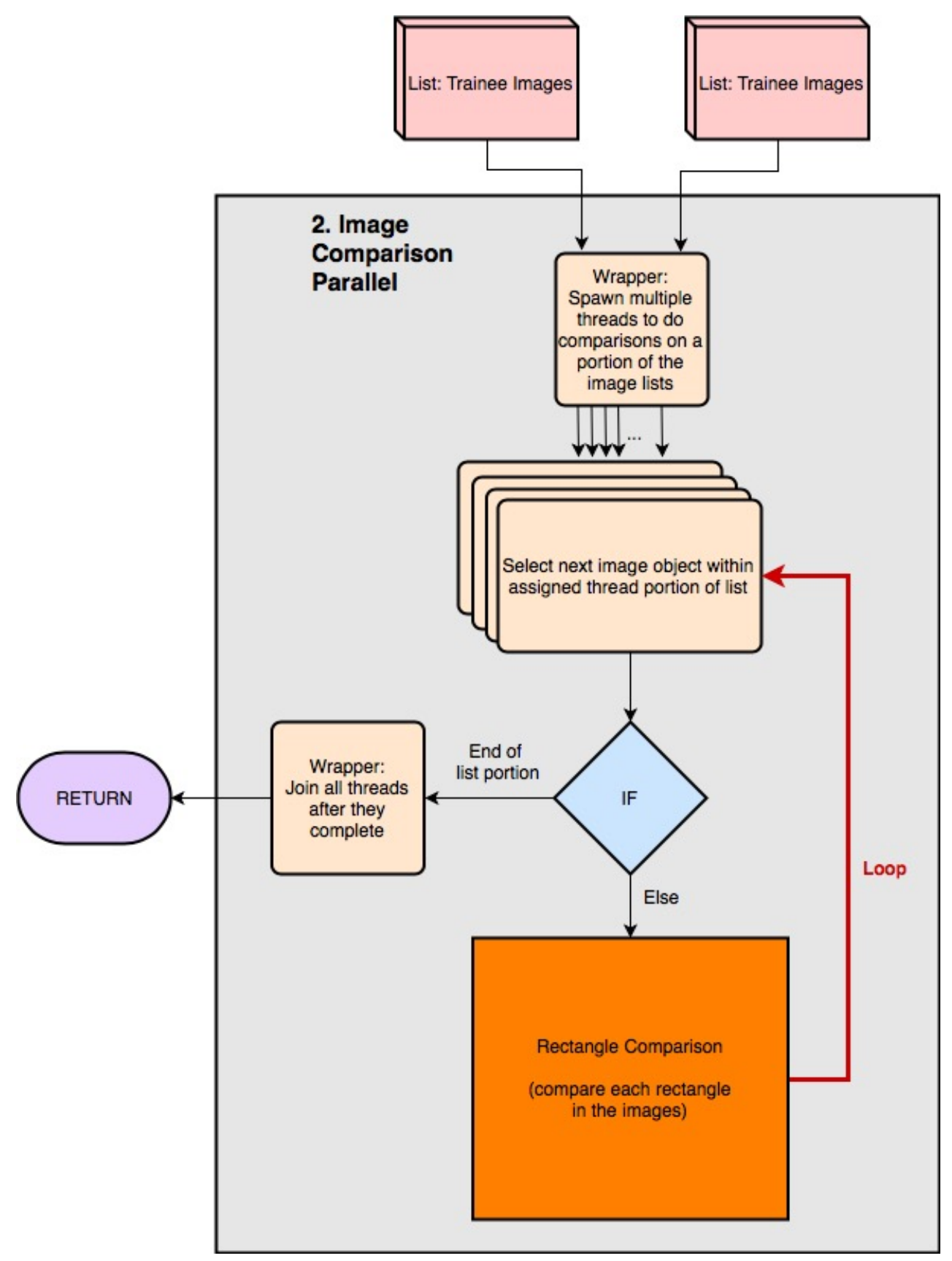

Figure 4.7: Flow diagram for imageCompParallel function 
2. imageCompareParallel

The imageCompareParallel function is the function that is run on each thread in the Parallel Image Comparison system. Figure 4.7 shows the program flow for the imageCompareParallel and wrapper functions. This function has four inputs: the vector for expert images, the vector for trainee images, the total number of threads, and the thread ID. Using the number of threads and thread ID, the function calculates the chunk size and position on which it is meant to conduct comparison. The function then conducts image-to-image comparisons on its chunk of the Image vectors. The rectangleCompareSerial function (see above) is used to compare the images in this method.

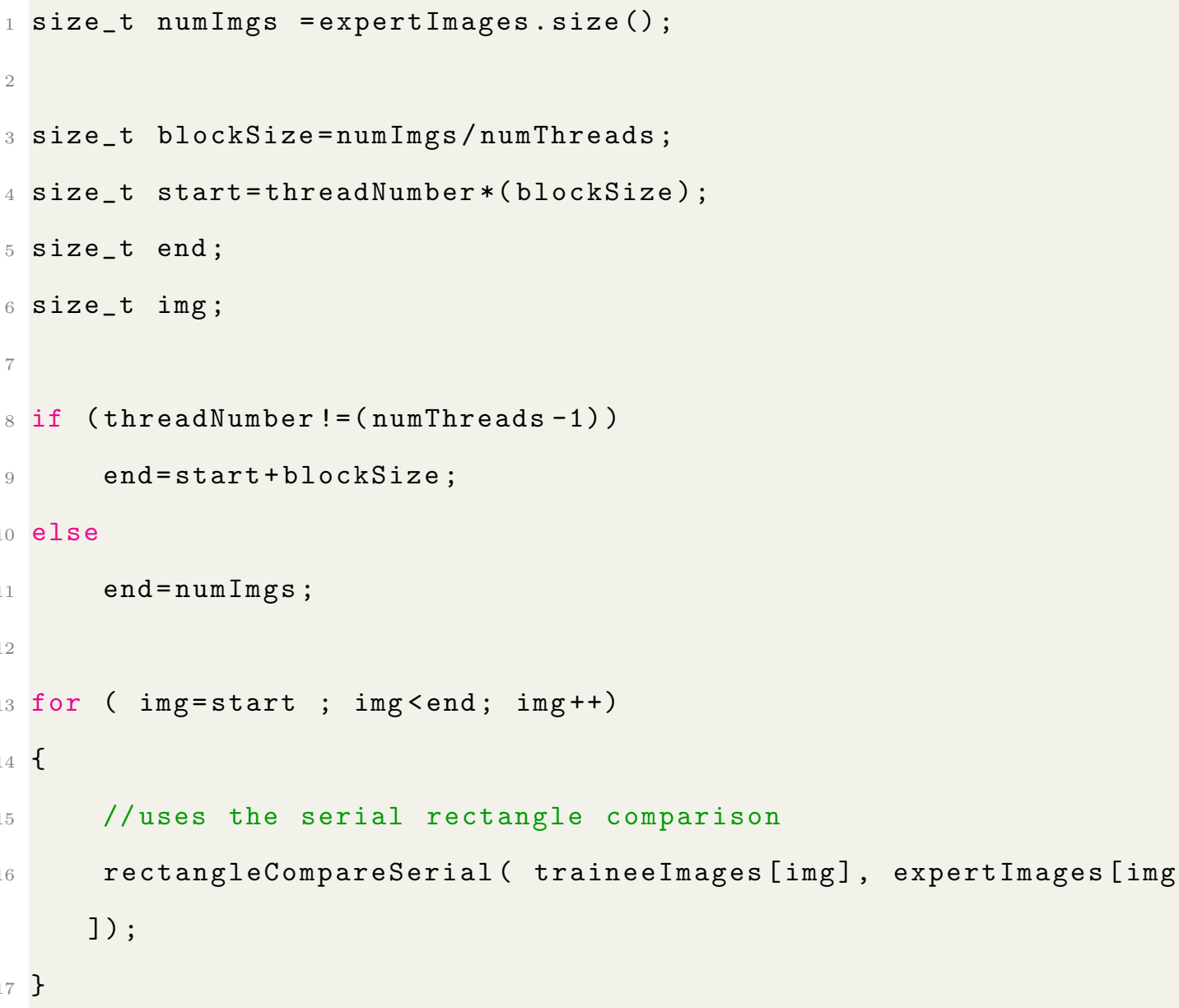

Once the imageCompareParallelWrapper function returns, the expert Images vector 


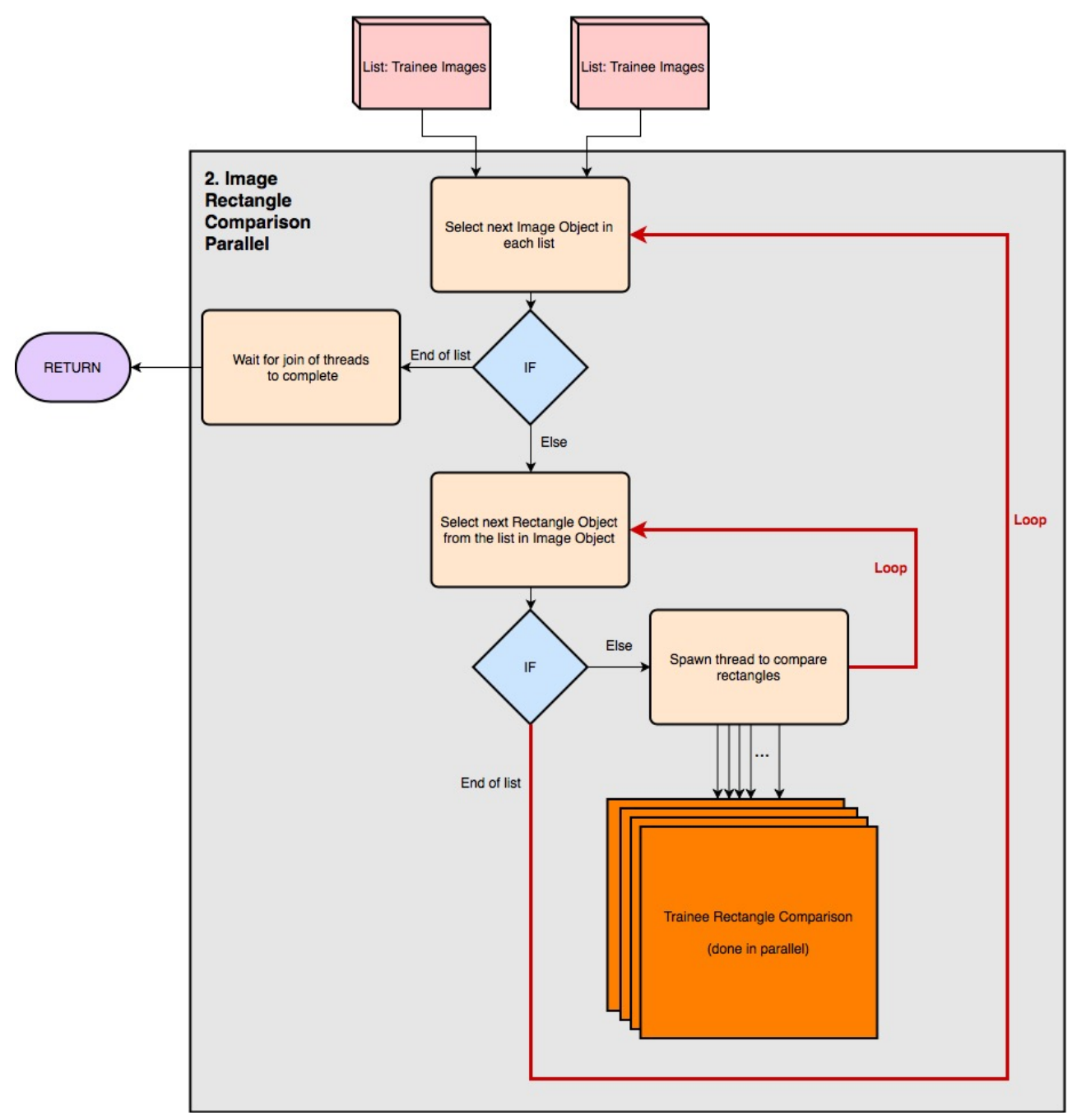

Figure 4.8: Flow diagram for rectangleCompareParallel function

contains scores for each image which can then be output into a text file for the trainee to view.

\section{Parallel Rectangle Comparison}

The second parallel comparison method targets the rectangle-to-rectangle portion of comparison for parallelization. This is a good method for parallelization when many cores are available to do a small amount of work.

1. rectangleCompareParallel 
The rectangleCompareParallel function has two inputs: a list of pointers to Images from the expert's solutions, and a list of pointers to Images from the trainee's solutions. Figure 4.8 shows program flow for this function. A for loop is used to select each Image Object in both the trainee and expert list of Image Objects. A second for loop is used to select each Rectangle Object in the expert Image. For each of these Rectangles, a thread is spawned that runs the traineeRectangleCompare function (see above), with the expert Rectangle and the trainee image as inputs.

Once all of the threads have been spawned and completed execution, the function joins all threads using a while loop.

Lastly, all of the expert Rectangles' scores must be added to the expert Images' total scores. This is done by iterating through each rectangle in each expert Image Object and adding the scores to the running total for the Images.

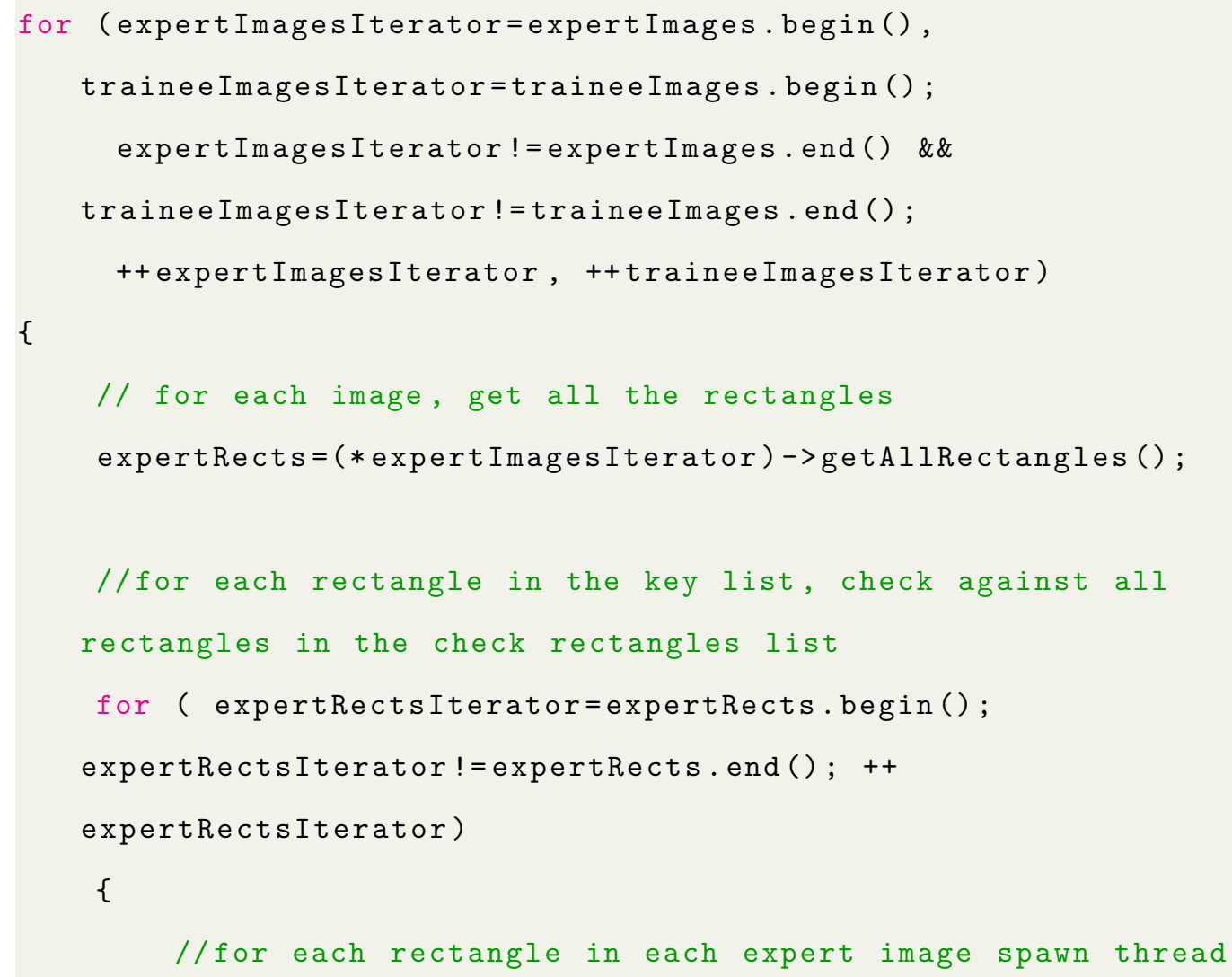




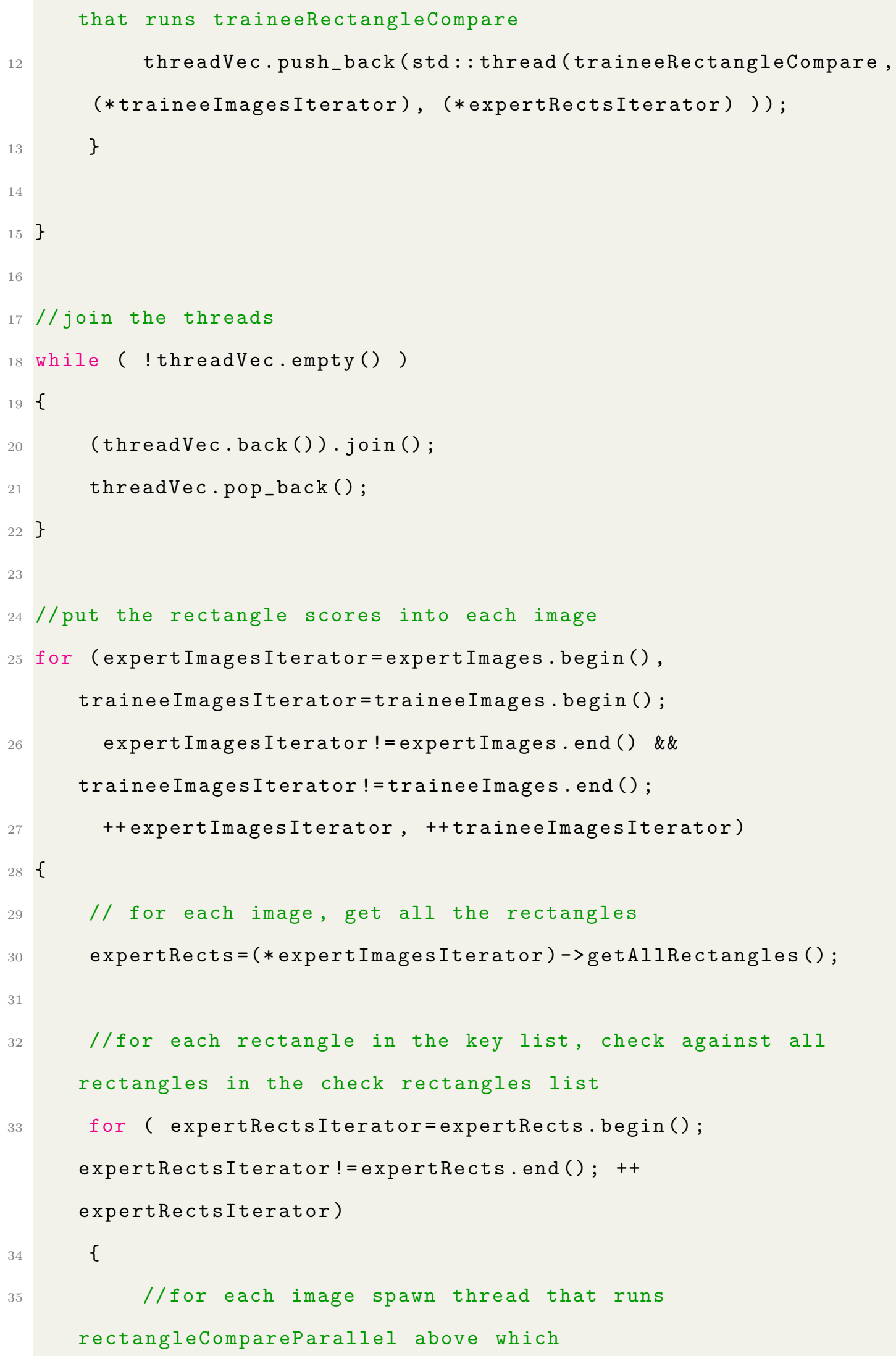




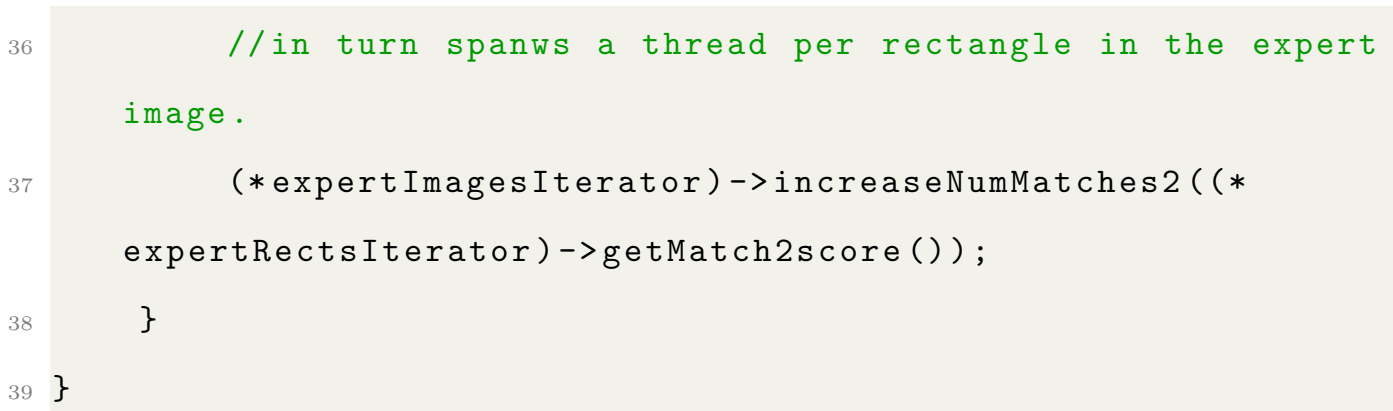

Once the rectangleCompareParallel function returns, the expert Images list contains scores for each image which can then be output into a text file for the trainee to view.

\section{Parallel File Comparison}

The third parallel comparison method targets file-to-file comparison for parallelization. This parallel method is used for situations where many files need to be compared to a single expert file at once.

\section{1. multiFileCompare}

The multiFileCompare function has four input arguments: a standard string denoting the path to the input files, an integer representing the threadID, an integer representing the number of threads, and an integer representing the number of files to be read in. Based on the threadId, number of threads, and number of files to be read, the multiFileCompare function determines which files to compare, conducts the comparison using the imageCompareSerial function for each file in sequence, and outputs the results file for each input file.

Provided below is the main logic of the function. The section of files must first be computed. The expert's data must be read in. Then a for loop is used to select each trainee file from the directory provided by the file path. The trainee data is read in and the comparison is conducted between the two 
image lists using the imageCompareSerial function. Results are output to the corresponding file. Lastly, the expert data must be cleaned to remove scores from the previous comparison and the trainee data must be deleted before looping again to select the next trainee file.

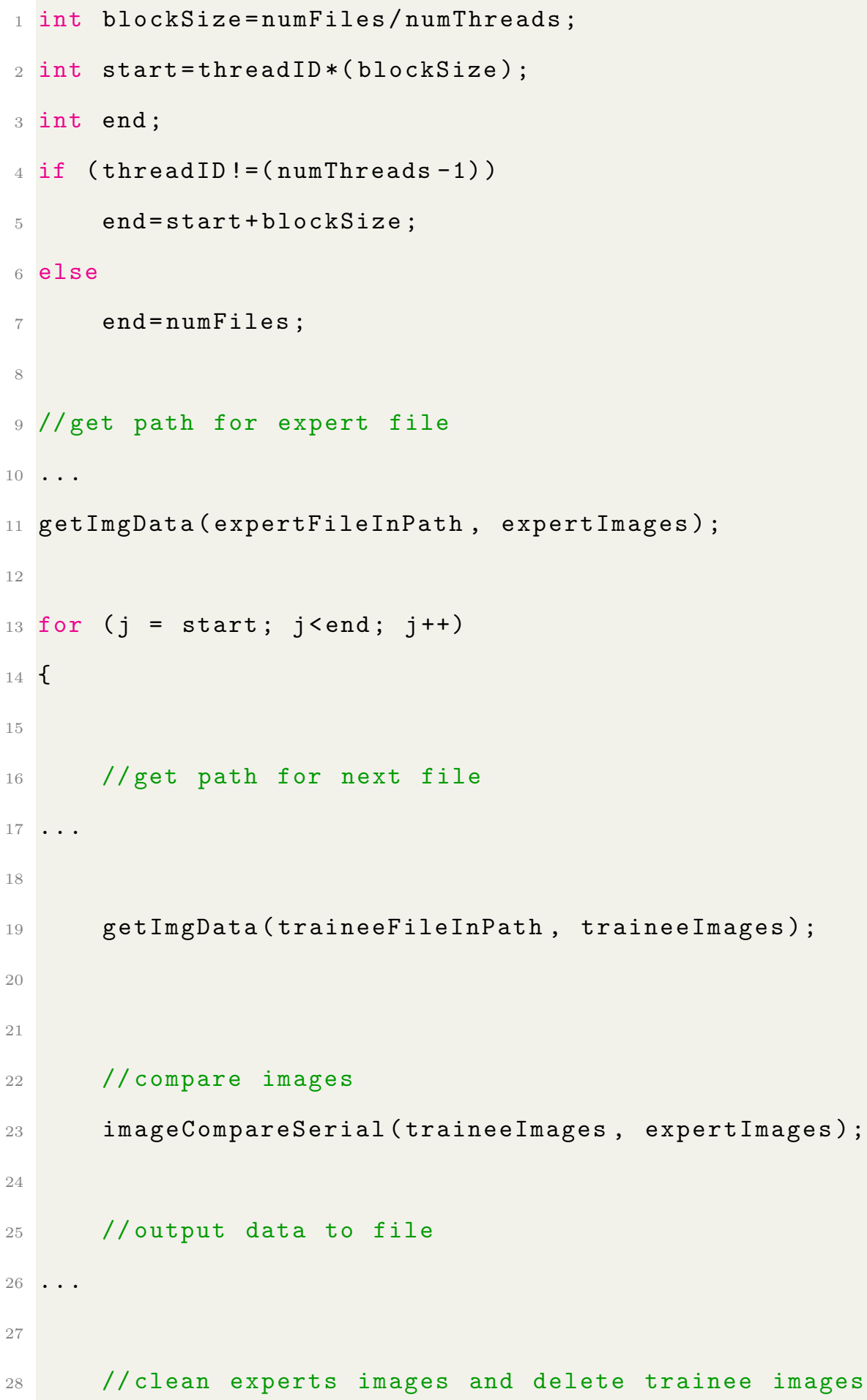




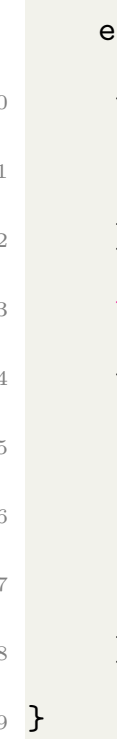

\subsubsection{Output Files}

Once the images have been compared in the lists and all scores are held in the list of expert Images, the output file must be generated. A for loop is used to iterate through each Image in the expert list. The score for the image is output to a text file as the total score out of the total number of rectangles the expert marked for that image. Further more this fractional value is used to compute a score for the entire set of images. Each image's score is converted to a value between 0 and 1, and then summed to give a submission-wide score out of the number of images. This score is also given as a percentage. Example output is bellow.

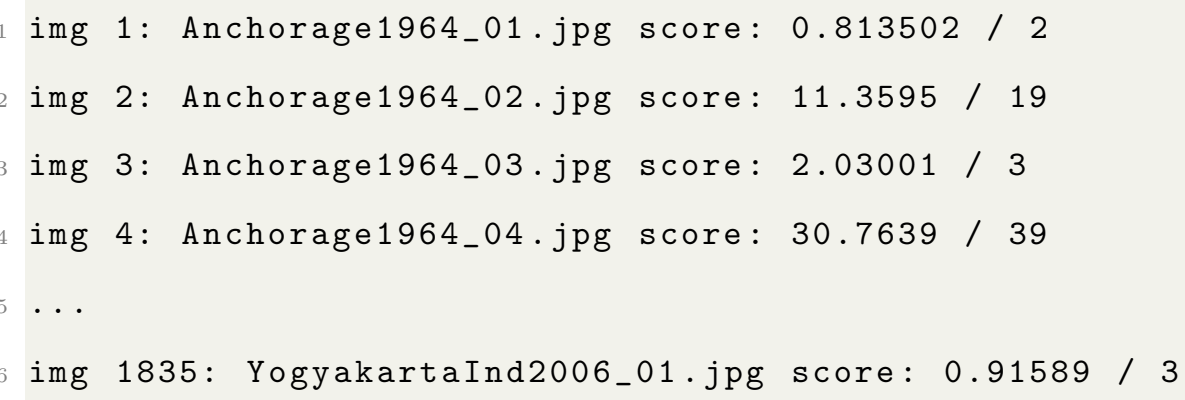




\footnotetext{
img 1836: YogyakartaInd2006_02.jpg score: 1.45907 / 3

8 The total score for the submission is: $774.449 / 1836$ or $42.1813 \%$
} 
Chapter 5

TESTING, EVALUATION, AND DISCUSSION

This chapter describes the evaluation criteria, testing approaches, and results for this project.

\section{$5.1 \quad$ Testing}

This section describes the different types of testing that were utilized to measure the efficacy of the final product of this thesis.

\subsubsection{Overview}

There are several different phases of testing that happened in this thesis to measure timing of different evaluation methods and to compare images tagged by different experts. The program conducts all tests in one run. Timing evaluation was broken up into two main sections: single-file and multi-file timings.

The first set of tests run are single-file timings where each of the three evaluation methods for single-file evaluation are run, and timing data is output for the tests. The goal of these tests is to determine the efficiency of the different methods for a single file. The three single-file evaluation methods are:

1. Serial comparison

2. Parallel comparison (image-to-image parallelization)

3. Parallel comparison (rectangle-to-rectangle parallelization)

Each of these evaluation methods compares the same expert and trainee files and a 
results file is output which includes scores for the comparisons and the amount of time taken to conduct the comparison.

The second set of tests run are multi-file timings where each of the four evaluation methods for multi-file evaluation are run, and timing data is output for the tests. The goal of these tests is to determine the efficiency of the different methods when a large number of files need to be compared. The four multi-file evaluation methods are:

1. Serial comparison

2. Parallel comparison (image-to-image parallelization)

3. Parallel comparison (rectangle-to-rectangle parallelization)

4. Parallel comparison (file-to-file parallelization)

Each of these evaluation methods compares a number of trainee files in a directory to a single expert file. Results for each file are output in the same directory. Additionally, the timing data for the multi-file evaluation is output to a report text file.

The third set of tests run are expert-to-expert file comparisons. The goal of these tests is to determine how closely different experts will mark the same images. To conduct this evaluation, serial comparison between each combination of experts' files is conducted, and a results file is output for each, detailing how similarly the experts marked the image.

\subsubsection{Testing Platform}

The majority of the tests used in this thesis to determine the efficiency and accuracy of the project were run on:

Model Name: MacBook Pro 
Model Identifier: MacBookPro11,1

Processor Name: Intel Core i5

Processor Speed: $2.8 \mathrm{GHz}$

Number of Processors: 1

Total Number of Cores: 2

L2 Cache (per Core): 256 KB

L3 Cache: $3 \mathrm{MB}$

Memory: 16 GB

Physical Drive:

Device Name: APPLE SSD SM0512F

Medium Type: SSD

Protocol: PCI

Internal: Yes

Additional tests were done on the high performance computers at Cal Poly. The test computer has 28 cores and $31 \mathrm{G}$ of memory.

\subsubsection{Data Files}

The input testing data files to this project are text files in the format provided by the graphical user interface described in Section 3.2. One expert file was provided at the beginning of this thesis, and five more were provided near the end of the project for testing purposes. The first file is the one that has been used for the primary timing and accuracy testing of this project, while the latter five have been used primarily for comparing evaluations that have been done by different experts. 


\subsubsection{Test File Generator}

In order to test the accuracy and speed of the work done in this thesis, some text files for testing needed to be generated from the first expert file provided by Dr. Pantoja. Additionally, since changes to the GUI, which would make the output text files have a single integer representing the damage/structure pair instead of a string, have not yet been made, the test files needed to be edited to conform to this requirement. These test files needed to meet several requirements:

1. Both the expert file and the trainee file needed to have the damage/structure pair tags converted to an enumeration that would be consistent across both files.

2. When generating the trainee files, the order of the rectangles in each image needed to be randomized. (The output file may have more or fewer rectangles than the original.)

3. When generating the trainee files, a small random variance for each rectangle's four edges needed to be included.

With these requirements in mind, a script to generate test text files based on the first expert file was used. This script was developed specifically for creating these test files by Mike Ryu. Mike Ryu is a computer science master's student at Cal Poly San Luis Obispo. He is also a personal friend and colleague of mine. The Python script which he developed for me for the purpose of generating test text files is provided in the appendix. The script takes in a text file and allows for the user to set several parameters that control the randomness of the output, the number of output files, and the configuration of the tag enumeration.

Once the test text files are generated, they can be used to test the speed and 
accuracy of the comparison tool. These text files simulate trainee submissions, since trainee submissions are expected to include:

1. Inaccurate rectangles which will match to some of the expert rectangles

2. Rectangles that will not be in the same order as those in the expert file

3. Either extra or missing rectangles

Using the generate data script, one trainee text file and one expert text file were created for testing. These files were further extended to include more images in a single file by duplicating the contents of the file. The resulting files each include 1836 images and a total 9336 rectangles for use in comparisons.

Additionally, another 499 unique test text files were created (each containing the original 153 images and 778 rectangles) for the four methods of multi-file evaluation. An exact copy of the expert file was also used as one of the tests to demonstrate that an identical file would receive a perfect score. The evaluation of these 500 text files could be timed to determine which methods were best. The test outputs a results file for each test text file, as well as an overall report on the evaluation for the method used.

\subsubsection{Timing}

The testing phase of this project utilized the dtime function to get accurate timing of the different evaluation methods. The dtime function was taken from labs provided in Dr. Pantoja's CPE 419 Parallel Computing class, which I took in Spring quarter 2017.

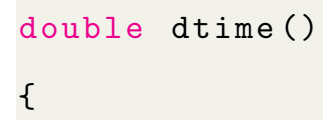


double tseconds $=0.0$;

struct timeval mytime;

gettimeofday (\&mytime, (struct timezone*)0);

tseconds $=\left(\right.$ double) $\left(m y t i m e \cdot t v_{-}\right.$sec + mytime.tv_usec*1.0e-6);

return ( tseconds );

\}

Timings were taken of:

1. Sequential method used on a single extended file. Evaluation was run 100 times to get an average time to evaluate. The extended files include 1836 images and a total 9336 rectangles.

2. Parallel method, parallelizing image-to-image comparison on a single extended file. Evaluation was run 100 times to get an average time to evaluate.

3. Parallel method, parallelizing rectangle-to-rectangle comparison on a single extended file. Evaluation run one time.

4. Sequential method used on the 500 generated test text files. A timing for the entire method and a separate timing for all but file output were taken for this method.

5. Parallel method, parallelizing image-to-image comparison on the 500 generated test text files. A timing for the entire method and separate a timing for all but file output were taken for this method.

6. Parallel method, parallelizing rectangle-to-rectangle comparison on the 500 generated test text files. A timing for the entire method and a separate timing for all but file output were taken for this method.

7. Parallel method, parallelizing file-to-file comparison on the 500 generated test 
text files. Only a timing for the entire method was taken for this method since File I/O was done in parallel for this method.

Additional timing tests were done on the high performance computer to determine if the parallel versions scaled with more cores. Timings were taken for the following methods with $2,4,8,16,28,32,64$, and 128 threads:

1. Sequential method used on a single extended file. Evaluation was run 100 times to get an average time to evaluate. The extended files include 1836 images and a total 9336 rectangles.

2. Parallel method, parallelizing image-to-image comparison on a single extended file. Evaluation was run 100 times to get an average time to evaluate.

3. Sequential method used on the 500 generated test text files, timing included the File I/O.

4. Parallel method, parallelizing file-to-file comparison on the 500 generated test text files, timing included File I/O.

Once timings have been taken of a method, the results are provided in a file. In the case of single file evaluation, timing data is provided in the same file as the scoring results. In the case of the multi-file evaluation, the timing data is output in a separate report file.

\subsubsection{Expert Files}

Late in the work for this thesis, five expert files were provided for testing purposes. These files came from various industry experts named Ezra, Ezra, Ana, Erika, and John. Two different people named Ezra provided expert submissions and the files 
provided are distinguished by numbering one Ezra as Ezra1 and the other as Ezra2. In order to determine how closely different experts mark the same images, each expert's file was compared to each of the other experts' files. In terms of the comparison this means that each file was treated as The Expert file and compared to the others as if they were The Trainee file. Since there were five files, this resulted in twenty comparisons. Furthermore, the experts' output files had metrics associated with the damage tags tagging the surety with which the expert had tagged the damage pair. These metrics were not able to be resolved to the enumerations required for comparing rectangles, so these experts' submissions were not compared using the damage tags. Instead, comparisons were conducted purely on rectangle matching using the Jaccard's index method.

Additionally, since the experts' submissions are not all of the same images, comparison was conducted only on images that had matching names, and two scores were computed. One score shows the total score of the trainee submission against the expert solution. The other is the total score for only the images in the trainee submission that were common in the expert solution. For example the Erika solution had only 12 images submitted and only 11 that were common, so when compared to the Ezra1 solution, the output looks like this:

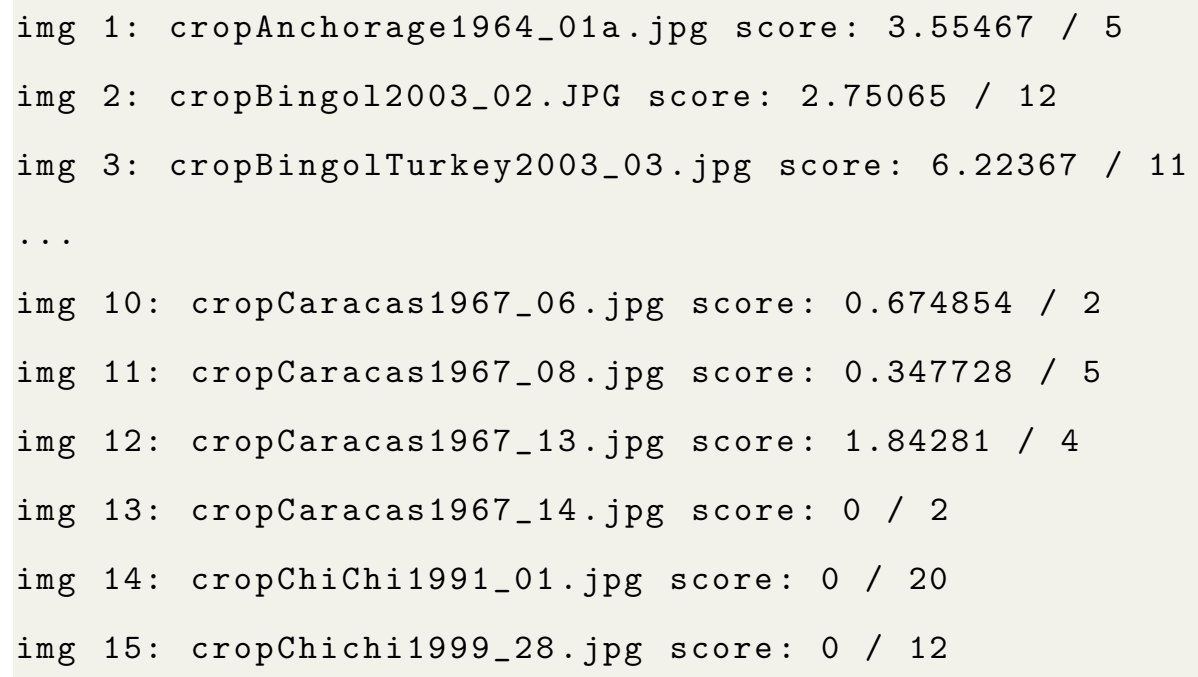




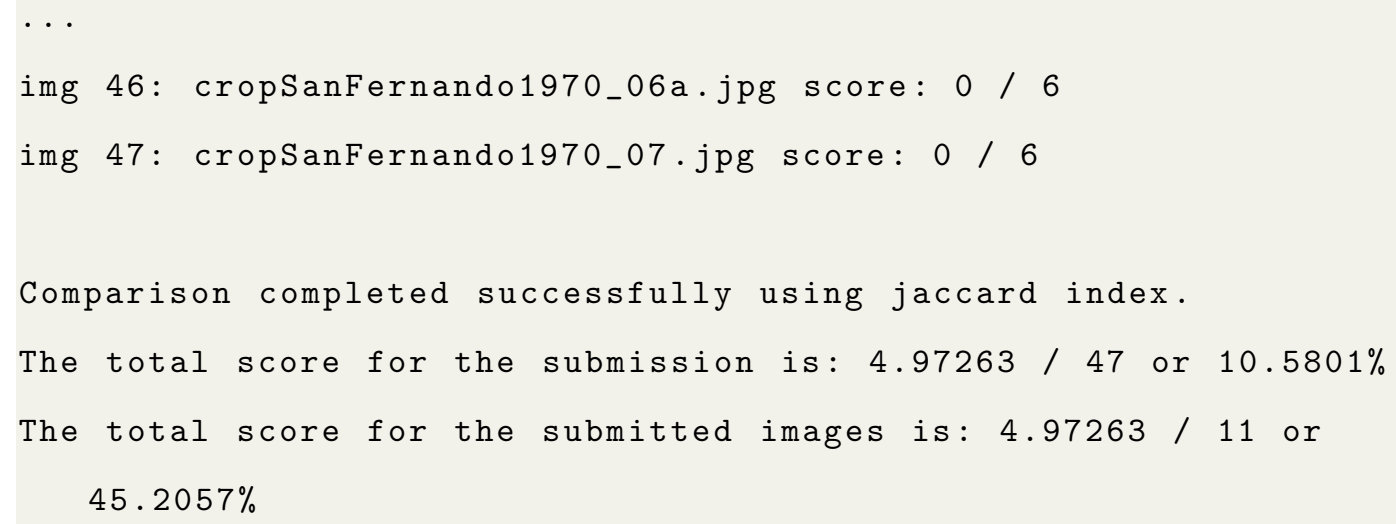

Results of each comparison were output to a results file detailing the scores for each image but also the overall score for the entire file. These results will help provide insight into what the ground truth solutions to each of these images really is.

\subsubsection{Profiling and Program Validation}

For this project, profiling and program validation were carried out using:

\section{Instruments}

Instruments is an analysis and profiling tool provided by Apple Inc. as part of their XCode Tool Set. Instruments is meant for profiling applications for better optimization and and understanding of the applications' behavior [2].

2. Valgrind

Valgrind is a tool used for detecting memory leaks, memory management and threading bugs, and detailed profiling of programs [9].

\subsection{Evaluation}

This section provides the results of the tests provided in the previous section. 


\subsubsection{Single-File Timings}

The timing results of comparing the single extended trainee file against the single extended expert file are given for the three evaluation methods provided below:

1. Serial comparison

Comparison completes in 0.0371819 seconds on average over 100 runs.

2. Parallel comparison (image-to-image 4 threads)

Comparison completes in 0.0167618 seconds on average over 100 runs.

3. Parallel comparison (rectangle-to-rectangle)

Comparison completes in approximately 15.0144 seconds.

\subsubsection{Multi-File Timings}

The timing results of comparing 500 trainee files against a single expert file are given for the four evaluation methods provided below:

1. Serial comparison

Comparison completes in approximately 2.85546 seconds when File I/O is included in the timing, or approximately 1.63095 seconds when File I/O is not included in the timing.

2. Parallel comparison (image-to-image 4 threads)

Comparison completes in approximately 2.14486 seconds when File I/O is included in the timing, or approximately 1.03409 seconds when File I/O is not included in the timing. 
Table 5.1: Results of High Performance Computer doing single file tests

\begin{tabular}{|l|l|l|l|l|l|l|l|l|}
\hline Threads: & 2 & 4 & 8 & 16 & 28 & 32 & 64 & 128 \\
\hline Serial: & 0.0202 & 0.0203 & 0.0205 & 0.0203 & 0.0206 & 0.0206 & 0.0204 & 0.0209 \\
\hline Img-Img: & 0.0157 & 0.0094 & 0.0049 & 0.0031 & 0.0032 & 0.00348 & 0.00419 & 0.0066 \\
\hline Speed up: & $1.28 \mathrm{x}$ & $2.15 \mathrm{x}$ & $4.18 \mathrm{x}$ & $6.35 \mathrm{x}$ & $6.32 \mathrm{x}$ & $5.93 \mathrm{x}$ & $4.88 \mathrm{x}$ & $3.13 \mathrm{x}$ \\
\hline
\end{tabular}

Table 5.2: Results of High Performance Computer doing multiple file tests

\begin{tabular}{|l|l|l|l|l|l|l|l|l|}
\hline Threads: & 2 & 4 & 8 & 16 & 28 & 32 & 64 & 128 \\
\hline Serial: & 1.2608 & 1.2652 & 1.2730 & 1.2647 & 1.2632 & 1.3394 & 1.2632 & 1.3223 \\
\hline File-File: & 0.6659 & 0.4736 & 0.2710 & 0.1692 & 0.1632 & 0.2855 & 0.4596 & 0.5830 \\
\hline Speed up: & $1.89 \mathrm{x}$ & $2.67 \mathrm{x}$ & $4.69 \mathrm{x}$ & $7.47 \mathrm{x}$ & $7.73 \mathrm{x}$ & $4.69 \mathrm{x}$ & $2.74 \mathrm{x}$ & $2.26 \mathrm{x}$ \\
\hline
\end{tabular}

3. Parallel comparison (rectangle-to-rectangle)

Comparison completes in approximately 25.7283 seconds when File I/O is included in the timing, or approximately 24.4965 seconds when File I/O is not included in the timing.

4. Parallel comparison (file-to-file 4 threads)

Comparison completes in approximately 1.45302 seconds when File I/O is included in the timing. A second timing is not taken for this method.

\subsubsection{High Performance Computer Timings}

For the results of the high performance computer running single file tests, see Table 5.1. Figure 5.1 shows the time to evaluate plotted against the number of threads.

for the results of the high performance computer running multiple file tests see Table 5.2. Figure 5.2 shows the time to evaluate plotted against the number of threads. 


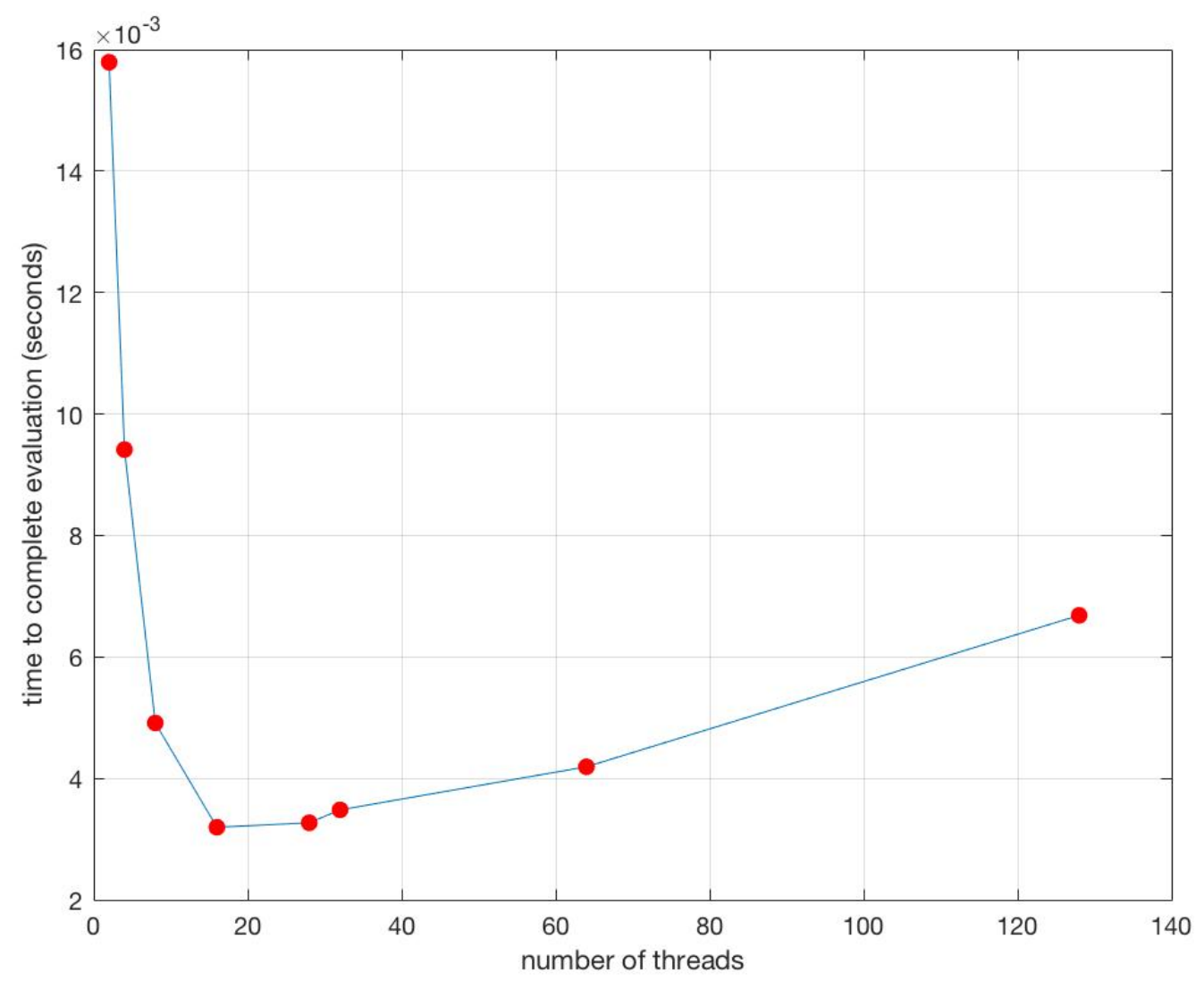

Figure 5.1: Results of High Performance Computer timing evaluations of Single File comparisons plotted against the number of threads used. 


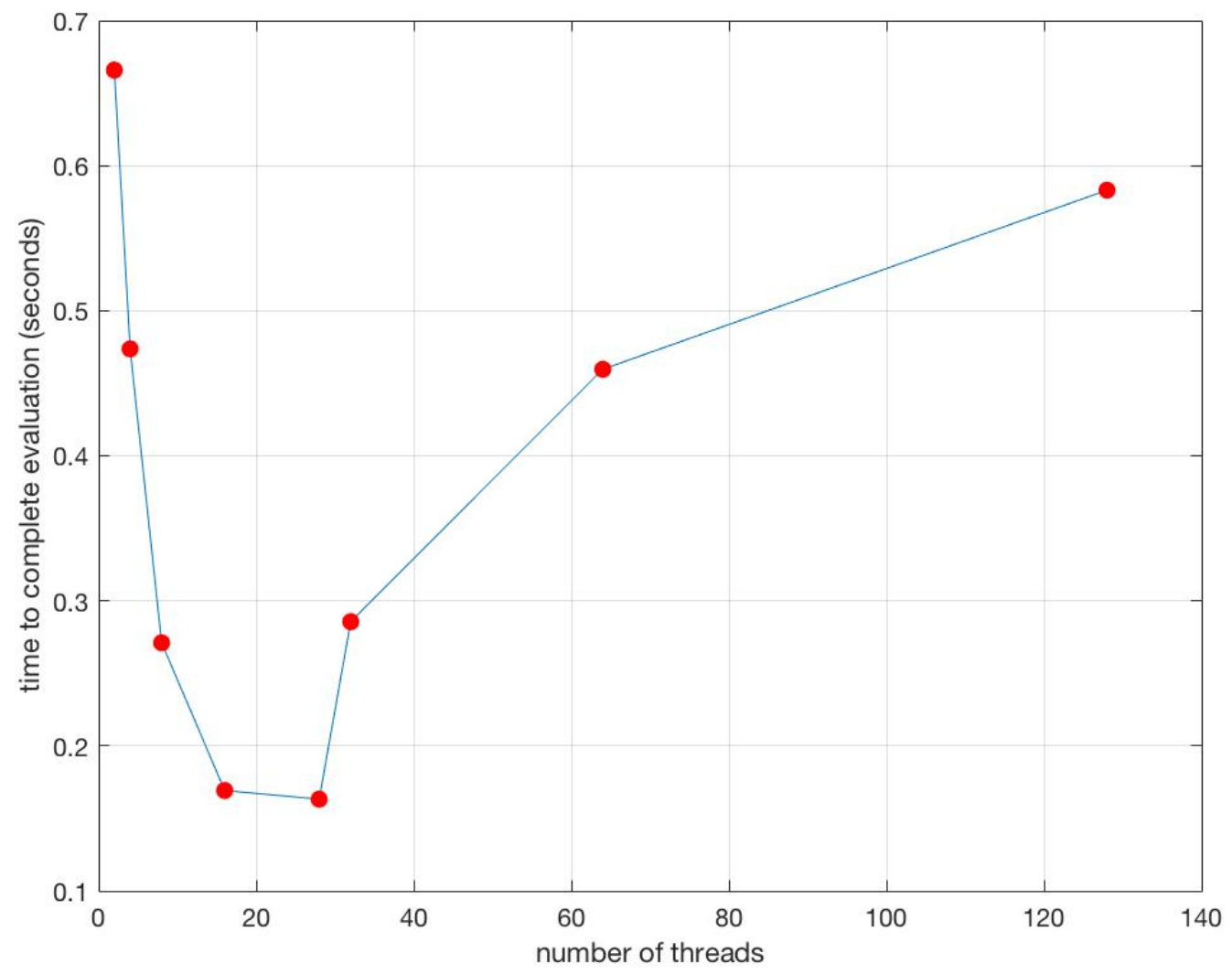

Figure 5.2: Results of High Performance Computer timing evaluations of Multiple File comparisons plotted against the number of threads used. 
Table 5.3: Results using Ezra1's file as expert

\begin{tabular}{|l|lll|ll|}
\hline \multicolumn{5}{|c|}{ Expert: 1. Ezra1 } \\
\hline Trainee & \multicolumn{2}{|c|}{ Total Scores } & \multicolumn{2}{|c|}{ Common Images Scores } \\
\hline 2. Ezra2 & $26.5771 / 47$ & $56.547 \%$ & $26.5771 / 47$ & $56.547 \%$ \\
\hline 3. Ana & $16.2578 / 47$ & $34.5911 \%$ & $16.2578 / 46$ & $35.3431 \%$ \\
\hline 4. Erika & $4.78146 / 47$ & $10.1733 \%$ & $4.78146 / 11$ & $43.4679 \%$ \\
\hline 5. John & $19.7628 / 47$ & $42.0486 \%$ & $19.7628 / 45$ & $43.9174 \%$ \\
\hline
\end{tabular}

\subsubsection{Expert File Comparisons}

The scores of image files as described in Section 4.2.3 are provided below for comparison between the files tagged by industry experts. Each table shows which file was used as The Expert and the scores of each other file used as The Trainee submission.

1. For results of comparisons using Ezra1 as the expert file, see Table 5.3

2. For results of comparisons using Ezra2 as the expert file, see Table 5.4

3. For results of comparisons using Ana as the expert file, see Table 5.5

4. For results of comparisons using Erika as the expert file, see Table 5.6

5. For results of comparisons using John as the expert file, see Table 5.7

Copies of the full reports created by doing each comparison are provided in the Appendix.

\subsubsection{Profiling and Program Validation}

The profiling results of Instruments are provided in Figure 5.3 and Figure 5.4. The

first profile, Figure 5.3, shows what functions were utilizing what percent of the full 
Table 5.4: Results using Ezra2's file as expert

\begin{tabular}{|l|lll|ll|}
\hline \multicolumn{5}{|c|}{ Expert: 2. Ezra2 } \\
\hline Trainee & \multicolumn{2}{|c|}{ Total Scores } & Common Images Scores \\
\hline 1. Ezra1 & $28.0546 / 47$ & $59.6907 \%$ & $28.0546 / 47$ & $59.6907 \%$ \\
\hline 3. Ana & $16.1555 / 47$ & $34.3733 \%$ & $16.1555 / 46$ & $35.1206 \%$ \\
\hline 4. Erika & $4.97263 / 47$ & $10.5801 \%$ & $4.97263 / 11$ & $45.2057 \%$ \\
\hline 5. John & $20.1657 / 47$ & $42.9058 \%$ & $20.1657 / 45$ & $44.8128 \%$ \\
\hline
\end{tabular}

Table 5.5: Results using Ana's file as expert

\begin{tabular}{|l|lllll|}
\hline \multicolumn{5}{|c|}{ Expert: 3. Ana } \\
\hline Trainee & \multicolumn{2}{|c|}{ Total Scores } & Common Images Scores \\
\hline 1. Ezra1 & $32.3502 / 51$ & $63.4319 \%$ & $32.3502 / 51$ & $63.4319 \%$ \\
\hline 2. Ezra2 & $31.5793 / 51$ & $61.9202 \%$ & $31.5793 / 51$ & $61.9202 \%$ \\
\hline 4. Erika & $6.5484 / 51$ & $12.84 \%$ & $6.5484 / 12$ & $54.57 \%$ \\
\hline 5. John & $28.5217 / 51$ & $55.9249 \%$ & $28.5217 / 49$ & $58.2076 \%$ \\
\hline
\end{tabular}

Table 5.6: Results using Erika's file as expert

\begin{tabular}{|l|ll|ll|}
\hline \multicolumn{5}{|c|}{ Expert: 4. Erika } \\
\hline Trainee & \multicolumn{2}{|c|}{ Total Scores } & Common Images Scores \\
\hline 1. Ezra1 & $7.57595 / 12$ & $63.1329 \%$ & $7.57595 / 12$ & $63.1329 \%$ \\
\hline 2. Ezra2 & $8.03102 / 12$ & $66.9252 \%$ & $8.03102 / 12$ & $66.9252 \%$ \\
\hline 3. Ana & $5.28157 / 12$ & $44.0131 \%$ & $5.28157 / 12$ & $44.0131 \%$ \\
\hline 5. John & $5.53065 / 12$ & $46.0887 \%$ & $5.53065 / 10$ & $55.3065 \%$ \\
\hline
\end{tabular}


Table 5.7: Results using John's file as expert

\begin{tabular}{|l|lll|ll|}
\hline \multicolumn{5}{|c|}{ Expert: 5. John } \\
\hline Trainee & \multicolumn{2}{|c|}{ Total Scores } & \multicolumn{2}{l|}{ Common Images Scores } \\
\hline 1. Ezra1 & $30.1703 / 45$ & $67.0452 \%$ & $30.1703 / 45$ & $67.0452 \%$ \\
\hline 2. Ezra2 & $28.0376 / 45$ & $62.3059 \%$ & $28.0376 / 45$ & $62.3059 \%$ \\
\hline 3. Ana & $21.8383 / 45$ & $48.5295 \%$ & $21.8383 / 44$ & $49.6324 \%$ \\
\hline 4. Erika & $5.41616 / 45$ & $12.0359 \%$ & $5.41616 / 9$ & $60.1796 \%$ \\
\hline
\end{tabular}

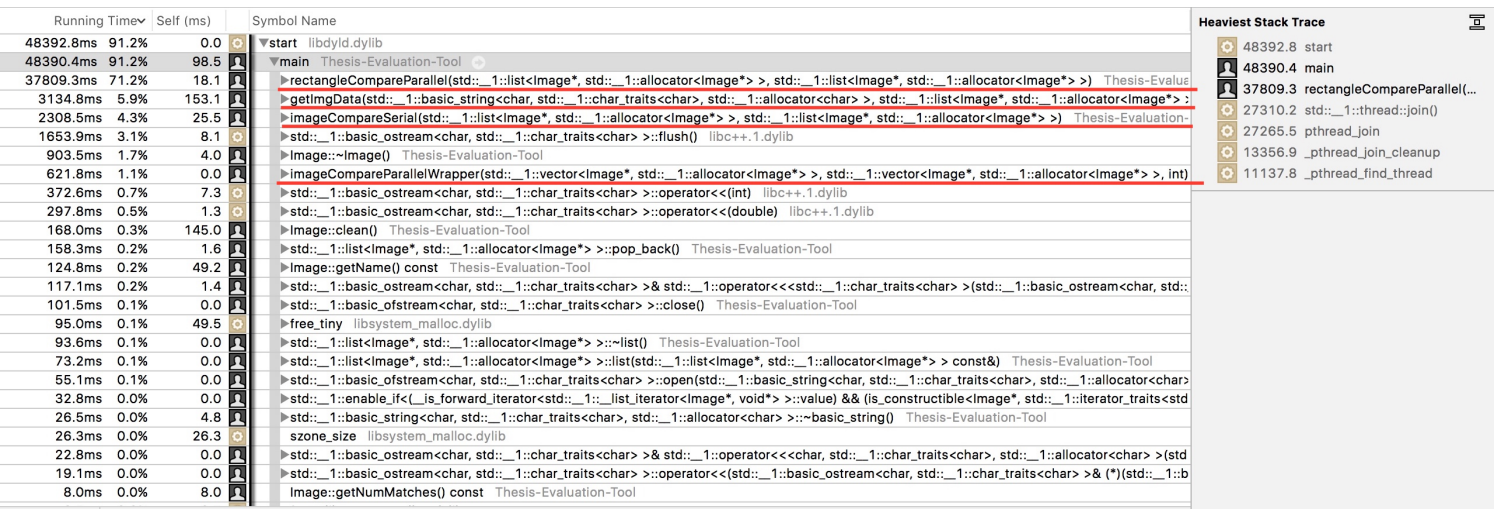

\section{Figure 5.3: Execution profiling INCLUDING the rectangle-to-rectangle parallel portions. Items of interest underlined in red.}

execution time. The second profile, Figure 5.4, shows the percent of execution time when the rectangle-to-rectangle parallel portions of execution are left out. High profile items are underlined in red.

Valgrind was used to check for memory leaks and showed concern about leaks in the getImgData function which reads data from the file and puts it into a list of Image Objects. There were possible leaks when a new Image Object and a new Rectangle Object were created in this function as they were not being deleted. Further details about these leaks is provided at the end of the discussion section below. 


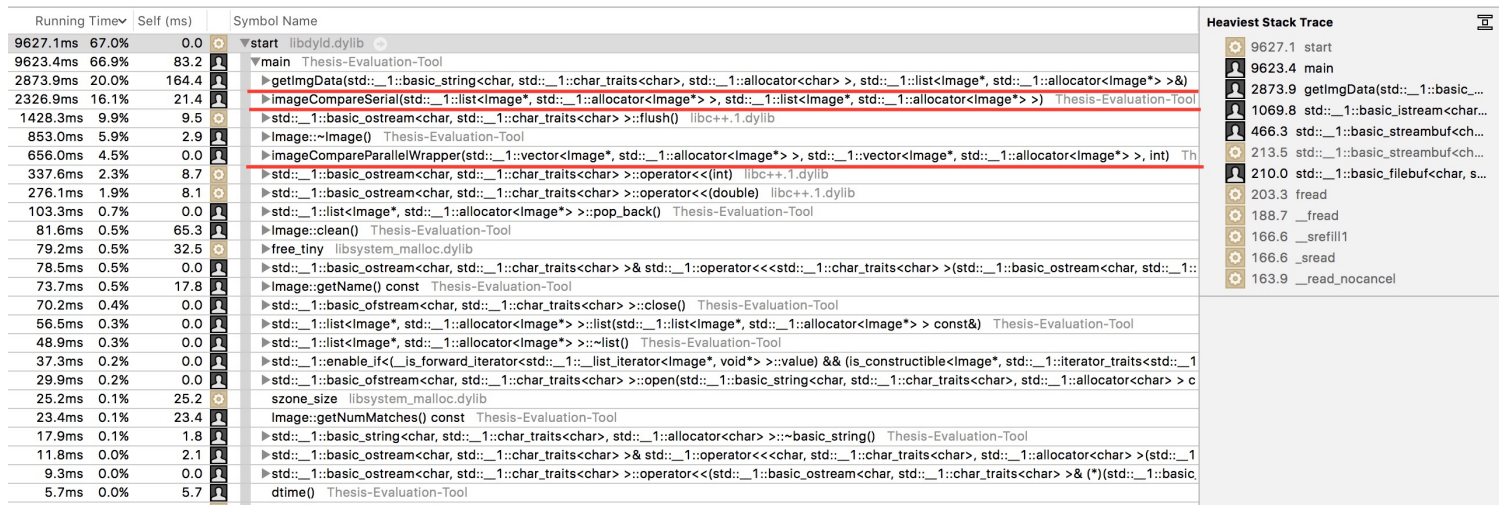

Figure 5.4: Execution profiling EXCLUDING the rectangle-to-rectangle parallel portions. Items of interest underlined in red.

\subsection{Discussion}

This section provides commentary on the results and significance of the tests detailed in the previous section.

\subsubsection{Single-File Timings}

For single-file timings, extended test text files were used to increase the amount of time the evaluation would take, since evaluations were completed in such small fractions of a second for the original length test text file that it was difficult to compare the different evaluation methods. However, even with the extended files containing 1836 images and a total 9336 rectangles, comparison was rather fast for two of the three single-file comparison methods. An average of 100 runs were taken to determine just how quickly these different methods complete.

\section{Serial comparison}

The serial comparison method was found to be quite fast despite utilizing the brute force approach to comparing rectangles in each image. Comparisons completed in under four one-hundredths of a second on average, making this a very feasible method for comparing a single-file. 
2. Parallel comparison (image-to-image)

The parallel comparison method, which parallelized the image-to-image portion of comparison, was largely successful. Acceleration over the serial comparison method was evident with evaluation completing in under two one-hundredths of a second on average. This is more than $2 \mathrm{x}$ faster than the serial comparison method. Although four threads were used for this evaluation technique, it is likely that the $2 \mathrm{x}$ speed up is due to the testing computer only having two cores. Despite the promising nature of this parallel method, for single-file comparisons, parallelization seems to be a bit overkill, as the serial comparison method is already very fast even for an extended file.

3. Parallel comparison (rectangle-to-rectangle)

The parallel comparison method, which parallelized the rectangle-to-rectangle portion of comparison, was largely unsuccessful. This method creates a thread per rectangle in the expert file, which means that there are a lot of threads trying to run at once. The overhead involved in spawning all of these threads was too much for the testing computer, which has only two cores, and this resulted in this method taking far longer than the other two. Since each time this method was run it took around 15 seconds, taking an average was deemed unnecessary.

The fact that this method took far longer to evaluate the test files than the other two methods took, it was not without merit. Methods for improving this version are further explored in Chapter 7, Future Work.

Overall, the single-file tests were successful. All versions provide the same, accurate feedback. While the rectangle-to-rectangle parallelized version was slow, the other two versions showed that these methods could be implemented very easily for single file evaluations providing immediate feedback. These methods would most 
likely lend themselves to a webinar/training program for people interested in learning to tag these images, as a trainee could complete the example problems, submit, and have evaluation take place between their submission and the expert's solutions immediately, with feedback being provided at the moment of submission.

\subsubsection{Multi-File Timings}

For multi-file timings, files of the same length as the original test text file were generated to simulate a situation where many files had been submitted to a server which needed to then evaluate and provide feedback. For this situation, four different evaluation methods were used. Two different timings for the multi-file evaluation methods were provided to differentiate the amount of time the comparison algorithms were taking from the amount of time it was taking to read and write 500 files.

\section{Serial comparison}

The serial comparison method was again found to be quite fast, despite being a brute force approach. This method completed evaluation in under 3 seconds when including the file $\mathrm{I} / \mathrm{O}$ in the timing, and about half that time was the algorithm conducting comparisons, while the other half was writing to files. While this is no longer instantaneous feedback, it is expected that if many files are being submitted to a server for evaluation all at once, immediate feedback is no longer expected, and 3 seconds of evaluation means that this is a practical solution.

2. Parallel comparison (image-to-image)

The image-to-image parallel method was once again found to be faster than the serial comparison method, completing evaluation in just over 2 seconds, with almost exactly 1 second of that time accounting for the algorithm conducting 
comparisons, while the rest can be contributed to file I/O. This method shows an acceleration of comparison completing 1.5x faster than the serial comparison method, and the full evaluation completing 1.3x faster than the serial comparison. These results are not as promising as the results of the single-file test, however, this is likely due to the overhead involved in spawning and joining threads for each file being compared.

\section{Parallel Comparison (file-to-file)}

The file-to-file parallel method parallelized the file-to-file portion of comparison. Since file I/O operations were occurring in parallel for this method, only one timing was taken for the evaluation of the entire directory of files. This method was found to be the fastest of the four methods used for multi-file evaluations. Completing 500 file comparisons in under 1.5 seconds, this method is $2 \mathrm{x}$ as fast as the serial version and $1.3 \mathrm{x}$ as fast as the image-to-image parallel version. This acceleration is expected as there are two cores on the test computer, and since file I/O is conducted in parallel, the evaluation was expected to complete twice as fast.

\section{Parallel comparison (rectangle-to-rectangle)}

The rectangle-to-rectangle method was expectedly slower than the other methods used to evaluate multiple files. Similar to the version used for single-file evaluation, the rectangle-to-rectangle parallelization spawns too many threads for the two cores on the test computer. This version of comparison takes around 25 seconds to complete, due to the overhead involved in handling so many threads. Similarly to the single-file evaluation, improvements to this version could be made and will be covered in further detail in Chapter 7.

Overall, the multi-file tests were successful. All versions provide the same, accurate feedback. While the rectangle-to-rectangle parallelized version was slow, the 
other three versions showed that these methods could be implemented very easily for multiple file evaluations providing fast feedback. These methods are expected to be used for situations where many files need to be evaluated at once. An example would be for neural network training.

\subsubsection{High Performance Computer Timings}

The timings taken on the High Performance Computer demonstrated the scaling of the best parallel versions with more cores. For single file comparisons, speeds of up to $6.35 \mathrm{x}$ faster than the serial version were attained. For multiple file comparisons, speeds of up to $7.73 \mathrm{x}$ faster than the serial version were attained. This shows that with more cores, an even more significant acceleration can occur. How this relates to further work in this project is discussed in further detail in Chapter 7. One of the reasons that the file-to-file parallel version may start to see a cap in the speed ups is that there may be too many threads trying to do File I/O simultaneously.

\subsubsection{Expert File Comparisons}

In order to evaluate whether or not different experts are tagging the same images in the same way, five expert files were compared to each other. At first glance, the scores of these comparisons seem quite poor. This is in part due to a few things:

1. Not all experts evaluated the same images.

To handle this, a second score was computed only evaluating the images that were common to both experts' submissions.

2. Some experts marked more rectangles than others.

3. Experts make mistakes when marking the images. 
For example, one of the expert solutions had a rectangle with no area in one of the images. The top and bottom edge of the rectangle were on the same pixel coordinate. This meant that it was impossible to get a score for that rectangle, even if an identical file was used, that rectangle always received a score of 0 .

4. Jaccard's index is unforgiving of mistakes

Due to the way that Jaccard's index is calculated using areas of the rectangles, the score decreases exponentially as the overlapping area of the two rectangles decreases. Figure 5.5 shows two rectanlges of the same size and shape but with areas not overlapping by $\mathbf{6}$ pixels in the $\mathrm{X}$ direction and $\mathbf{6}$ pixels in the $\mathrm{Y}$ direction. These two rectangles appear to be matching pretty well but their Jaccard index score is only $\mathbf{0 . 6 2 5}$. Figure 5.6 shows how the Jaccard index score decreases as the overlap of the two rectangles in Figure 5.5 decreases.

Larger rectangles are more forgiving of this feature of Jaccard's index, however it is not perfect, and so scores of $60 \%$ or more for a rectangle are deemed to be pretty good matches.

Keeping these admissions about the tests on the experts' submissions in mind, the scores for the comparisons were still quite poor. The best score comes from comparing with John's solutions as the expert and Ezra's first submission as the trainee, in which a score of $67 \%$ was achieved with some submissions dipping down into the mid 30s. These poor scores are also partly linked to the fact that the experts are not marking the same number of rectangles on each image. This gives a strong indication that the experts are not agreeing on where to mark the images for damage, and these scores do not include the damage tag pairs. 


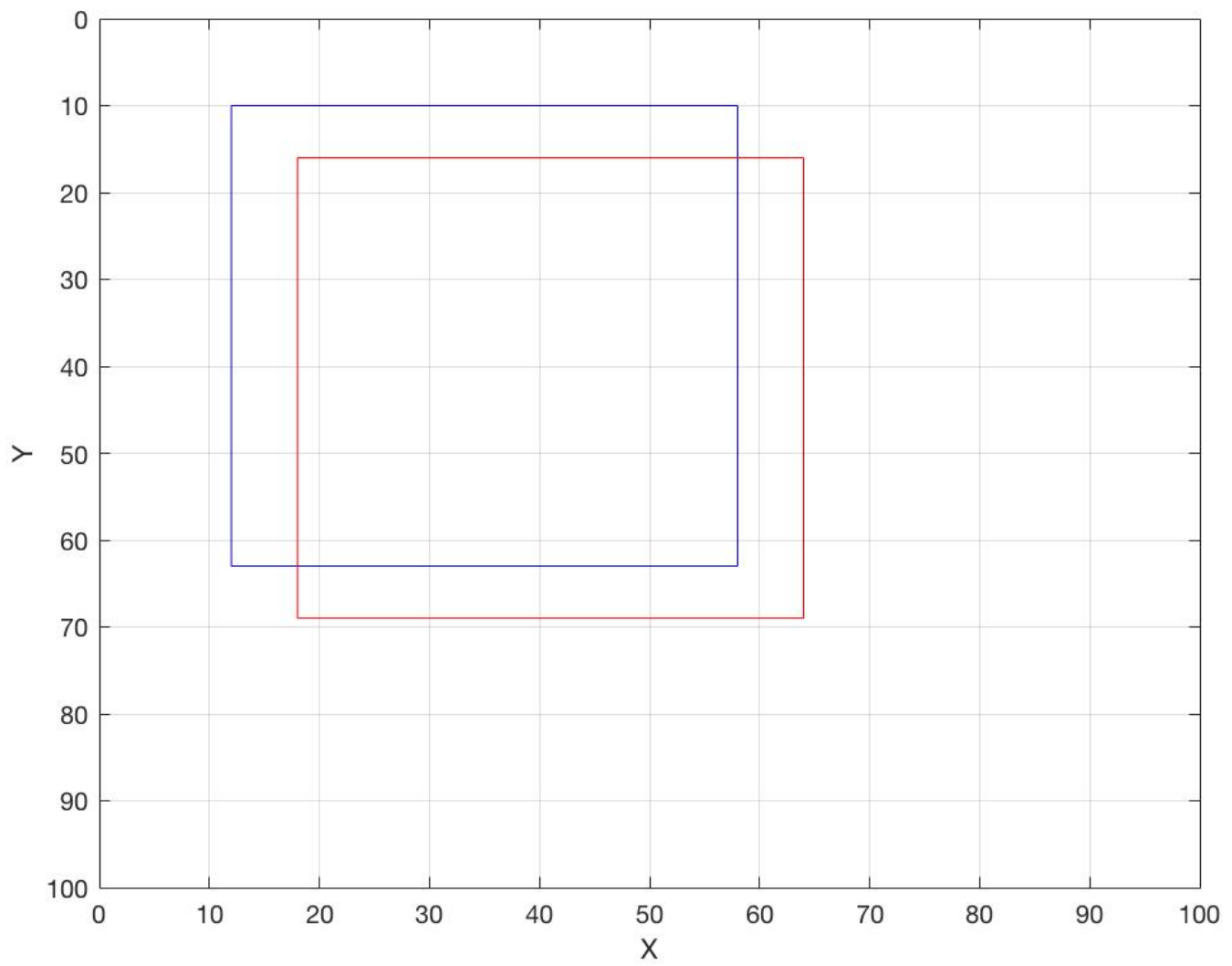

Figure 5.5: Two rectangles of the same size and shape offset by 6 pixels in the $\mathrm{X}$ and $\mathrm{Y}$ direction with a Jaccard's index score of 0.625 


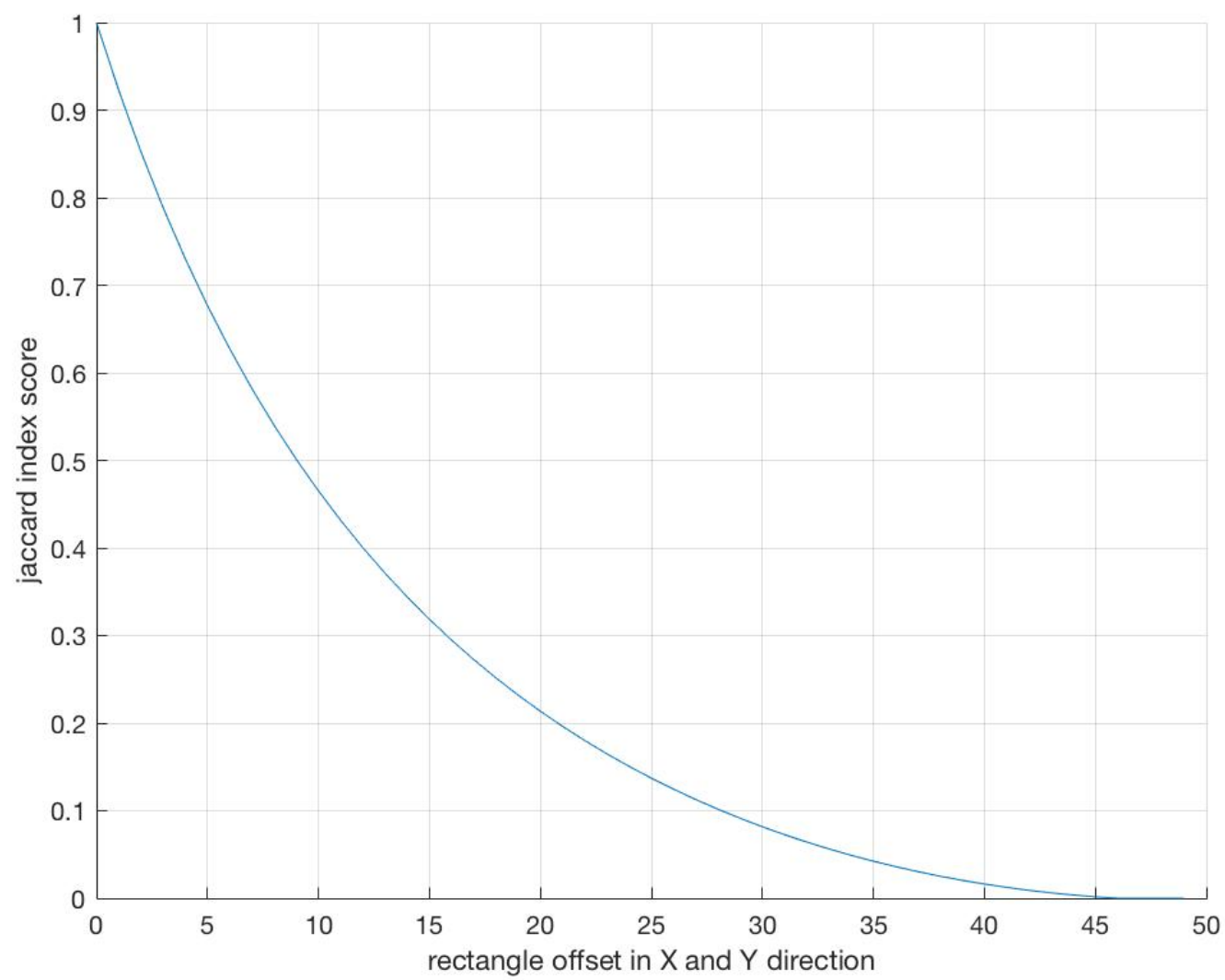

Figure 5.6: Jaccard's index scores as the overlap of the rectangles in figure 5.5 decreases. Note that the score falls off dramatically even for a small amount of misalignment 


\subsubsection{Profiling and Program Validation}

The profiling of the tests gave results that match with the timing results provided in the results Section 5.2. The rectangle-to-rectangle parallel method of comparison takes up the majority of CPU time (71\%), despite it only being run one time in the single-file version of the program.

When the overpowering rectangle-to-rectangle parallelization methods are left out of the test, the getImgData function takes up the most CPU time at 20\%. Serial comparison is a close second at $16 \%$ followed by file output, Image Object deletion from lists, and image-to-image parallel comparisons.

Valgrind provided information suggesting possible leaks were occurring in the getImgData function. Upon further inspection, Valgrind was finding that there were memory leaks when a new Image or Rectangle Object was created (using new) in this function because they were not deleted (using delete) before the function's completion. However, this is not a memory leak as the addresses for these objects were being pushed onto lists after being created. Therefore, no memory was being leaked in this function. 


\section{Chapter 6}

\section{CONCLUSION}

In this thesis an algorithm was successfully developed for quantifying how closely labeled areas on images are matching. This algorithm is very important to assign a measure of the quality and consistency of experts labeling images, a fundamental task for the development of machine learning algorithms. Several different methods for comparing files were developed and timed to show that different methods would be viable for different uses of the feedback system.

It was found that, for single-file comparisons, the serial version was sufficient even for very long files, completing in under 0.04 seconds. The parallel version, parallelizing the image-to-image portion of comparison further improved things with a $2 \mathrm{x}$ acceleration over the serial version.

For multi-file comparisons, the serial version was found to be sufficient for files numbering in the hundreds, with evaluation of all 500 test files completing in under 3 seconds. Further improvement of this method parallelized the file-to-file comparisons and improved the times to 1.5 seconds, an acceleration approximately $2 \mathrm{x}$ faster than the serial version.

While analyzing the expert files showed that the evaluations provided by experts present very important differences. Not only were the number of labels made different but also the types of damage associated with the label were vastly different between different experts. Some experts tagged the damage as catastrophic while others didn't label it at all or labeled it as less important. This finding lead to the conclusion that the experts tagging images for earthquake damages need to be trained in a standard way. This is a very important point, since the Pacific Earthquake Engi- 
neering Research Center at UC Berkeley is opening a challenge for summer 2018 [12], automatically label images after an earthquake and wants to create a 20000 images database to be used as the ground truth training set for the challenge. Evaluation of different experts' solutions found that, although they are tagging similar areas, further improvements need to be made before a good ground truth set of image solutions can be made for use in training.

Once the work in this thesis is implemented in the overarching project being conducted by Dr. Pantoja, the feedback system will be utilized to help trainees learn to tag images themselves in a post-earthquake scenario, vastly reducing the amount of time needed to determine which buildings are safe to use by those in affected areas. The feed back system will also be implemented as a way of training a neural network (NN) to do the same tagging, a long distance goal of the project of Dr. Pantoja. The unique form of feedback that this project provides will allow the NN to develop certainties about how likely a section of an image is to represent damage. 


\section{Chapter 7}

\section{FUTURE WORK}

Since the work in this thesis is a small part of a much larger project to not only create a way for non-expert civilians to take training courses on tagging images of post-earthquake infrastructure but also as a way to improve training of a neural network (NN) that could do the tagging, there is plenty of work to be done in the future. This chapter will cover some of the work that will utilize this thesis as well as some future improvements that could be made to this project to make it more useful in the overarching project.

The first improvements that can be made to this project are to use more powerful computers to conduct evaluations. The parallel versions that parallelize the image-to-image and file-to-file portions of comparison would lend themselves nicely to computers with more cores. Especially the file-to-file parallel comparison version would work nicely on a server that is being used to compare many files for training a neural network, for example. Potentially, tens of thousands of files would need to be compared for a situation such as this, and a super computer with many cores or a distributed system would work nicely for doing these evaluations, as a significant amount of file I/O would need to take place. Distribution using MPI would be a good fit here, as the file-to-file comparisons can occur asynchronously.

Along the same idea of using more powerful hardware for the comparisons, the rectangle-to-rectangle comparisons could be distributed across a graphical processing unit. This would likely make this parallel method instantaneous instead of taking longer than the serial version, as it does now. This is because this method lends itself very nicely to a solution where hundreds or thousands of threads can be running at the same time. The rectangle-to-rectangle comparison could be further improved to have 


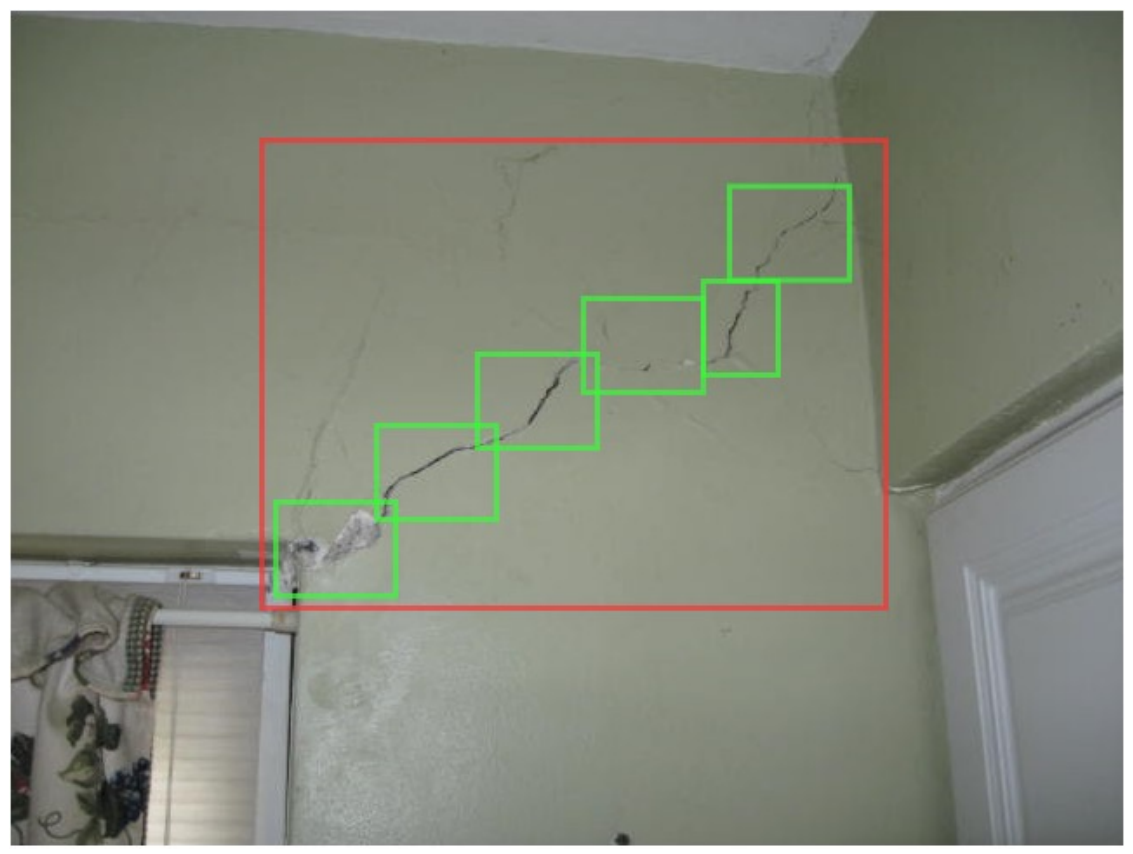

Figure 7.1: Example of two different ways to mark a diagonal crack in a wall. Which method is better and can both be used in future work?

each rectangle compared to each other rectangle in parallel, instead of the current implementation where each rectangle in the expert solution has its own thread for comparing to all the rectangles in the corresponding image of the trainee solution.

Scoring could be improved if a way to handle multiple rectangles being used to mark the same damage was accounted for and all rectangles counted towards that score. For example if there is a large damage area on a building like a crack that runs diagonally, is it better to draw a rectangle around the entire diagonal crack or would a few smaller rectangles be more appropriate. See Figure 7.1 for a visual example. If both options could be accounted for this would be an improvement to the versatility of the Fast Feedback Tool.

The output of the evaluations can definitely be improved in different ways for improving the feedback that trainees receive while learning to tag images or for the NN which, will eventually be using the outputs of this thesis to help it train. Currently, the output from this project is text files with scores for each image ranging from 
0 to the number of rectangles that the expert solution had for that image. Things could be further improved by giving more information about where each rectangle was meant to be. For example, a graphical output could be provided to trainees in which the expert's rectangles and the trainee's rectangles are overlaid on the image with some way of marking which rectangles matched and what score they received. This would help trainees to get a better understanding of which parts of the image were meant to be marked as apposed to the current blanket score out of number of expected rectangles for each image.

Feedback to trainees and the NN could be further improved by normalizing the values produced by Jaccard's index. Since there is a huge fall off involved with less overlap of rectangles, the scores could be better described, or some kind of multiplier could be used to better show when two rectangles are good matches.

Further analysis of the what types of infrastructure damage require which tags will definitely improve both the training of the $\mathrm{NN}$ as well as the process of people learning to tag images. If the tags can be consolidated, it means less memorization for people learning to do the tagging, and images required to train the NN. Much more hands-on work with experts in the field will be required to achieve this goal, since it has already been shown that the experts do not agree on where the rectangles even need to be. This leads into the need for experts to collaborate further on a ground truth set of solutions. Again, this would require more hands-on work with the experts in the field to get them to agree on what the ground truth of the solutions ought to be. This could also help to improve the feedback of this project, as the experts may have suggestions on how to improve the comparison method so that it better reflects when a correct solution has been provided as opposed to an incorrect solution.

There is a possibility for avoiding the need to create single ground truth files for each image which is obviously one of the major challenges of this project. This is a 
very important problem since getting that truth file requires confirmation from an infield team and in some cases that is impossible to get or very difficult. So comparing the quality of the experts without this file is important and requires the following:

1. Find the density functions: By assuming that each expert's assessment is independent of all the other expert's assessments. That is, the random variables $P\left[L_{j} \mid \Phi_{s}, E_{i}\right]$ are conditionally independent with respect to each expert.

2. Fourier transform of the density function: Calculate the density function of the sum of two independent random variables computed by finding the convolution product of the two density functions. The convolution integral may also be computed using a Fourier transform.

As stated previously, the work done in this thesis will be used in later work at Cal Poly. The feedback system created here can be incorporated into a training webinar that would allow for non-experts to learn how to tag images of damaged buildings in a post-earthquake scenario. The recommended method of integration would be to use the single-file serial comparison method to provide users with feedback on their submissions as soon as they submit. This method is fast enough for single-file evaluations and could be implemented in a way that it runs on the client-side of the webinar's platform to provide instant feedback to the user.

Also stated previously, the feedback system can be used to help train a NN for tagging images of building damage in a post-earthquake situation. It is likely that if a $\mathrm{NN}$ is being trained, then tens of thousands of images will be needing to be analyzed at once. The recommended method of integration for this situation would be to use the file-to-file parallel comparison method, either run on a super computer with many cores or run on a distributed system (possibly with MPI) to provide the feedback needed by the NN in a timely manner. 
This master thesis concentrated on accelerating the comparison of labeled regions on images. But further improvements can be made. Experts label regions of interest and assign a specific tag to it, and this process is prone to errors. In some cases, the errors can be catastrophic, for example the very well known case in which a self driving car classified an all white truck on a very bright day as a clear path and ended up killing the driver of the car. The problem in this case was that the NN was not trained with this situation and, instead of giving a result that indicated that it has never seen this before, since NN are basically forced to choose one of the output labels, it chose the most probable one. White usually means clear path. Our project tries to address this problem by providing the NN with the ability to indicate levels of uncertainty. The above example is similar to the case study for this thesis, in which images are taken after an earthquake. Since different experts give different labels it is clear that there is going to be uncertainty in the training set, and providing this information to the training of the NN not only will make it more accurate but also more reliable.

For the NN to output not just the value of the label but also the certainty, we needed to asses the quality of the experts and feed this information as an input to the NN. To evaluate the quality of the experts is of course paramount to get comparison of the labeled regions. 


\section{BIBLIOGRAPHY}

[1] Cal Poly Github. http://www.github.com/CalPoly.

[2] Apple_Inc. Instruments User Guide: About Instruments.

https://developer.apple.com/library/content/documentation/ DeveloperTools/Conceptual/InstrumentsUserGuide/index.html\#//apple_ ref/doc/uid/TP40004652-CH3-SW1.

[3] P. Bazzurro, C. Cornell, C. Menun, and M. Motahari. Guidelines for seismic assessment of damaged buildings. In Proceedings of the 13th World Conference on Earthquake Engineering, Vancouver, Canada, 2004.

[4] A. Behrouzi and M. Pantoja. Photo Tagging Tool for Rapid and Detailed Post-Earthquake Structural Damage Identification. 2018.

[5] P. Dabove, V. Di Pietra, and A. M. Lingua. Close range photogrammetry with tablet technology in post-earthquake scenario: Santagostino church in amatrice. GeoInformatica, pages 1-15, 2018.

[6] J. M. Del Rosario, R. Bordawekar, and A. Choudhary. Improved parallel i/o via a two-phase run-time access strategy. ACM SIGARCH Computer Architecture News, 21(5):31-38, 1993.

[7] K. Jobe. Disaster Relief in Post-Earthquake Haiti: Unintended Consequences of Humanitarian Volunteerism. Travel medicine and infectious disease, $9(1): 1-5,2011$.

[8] A. Kendall and Y. Gal. What uncertainties do we need in bayesian deep learning for computer vision? In Advances in Neural Information Processing Systems, pages 5580-5590, 2017. 
[9] N. Nethercote and J. Seward. Valgrind: a framework for heavyweight dynamic binary instrumentation. In ACM Sigplan notices, volume 42, pages 89-100. ACM, 2007.

[10] NHTSA. PE 16-007. Tesla Crash Preliminary Evaluation Report. In Technical report, U.S. Department of Transportation, National Highway Traffic Safety Administration, 2017.

[11] M. J. Olsen and R. Kayen. Post-earthquake and tsunami 3d laser scanning forensic investigations. In Forensic Engineering 2012: Gateway to a Safer Tomorrow, pages 477-486. 2013.

[12] Pacific Earthquake Engineering Research Center. PEER Hub Imagenet (PHI) Challenge Pre-Announcement, 2018.

[13] V. Powell and L. Lehe. Principal component analysis explained visually. DISQUS, Available: http://setosa. io/ev/principal-component-analysis/.[Accessed 11 July 2016], 2015.

[14] R. Real and J. M. Vargas. The probabilistic basis of jaccard's index of similarity. Systematic biology, 45(3):380-385, 1996.

[15] C. Rojahn. Procedures for postearthquake safety evaluation of buildings. Applied Technology Council, 1989.

[16] H. H. Ross. Principles of numerical taxonomy. Systematic Zoology, 13:106-108, 1964.

[17] C. M. Yeum, S. J. Dyke, and J. Ramirez. Visual data classification in post-event building reconnaissance. Engineering Structures, 155:16-24, 2018. 


\title{
APPENDICES
}

\author{
Appendix A \\ REPOSITORY
}

\begin{abstract}
All of the source, test files and related files to this project can be found at my Github repository for reference and usage:

https://github.com/MulliganKyle/Thesis-EvaluationTool
\end{abstract}

